\title{
VIBES: Visual Binary Exoplanet survey with SPHERE
}

\section{Upper limits on wide S-planet and S-BD frequencies, triple system discovery, and astrometric confirmation of 20 stellar binaries and three triple systems ${ }^{\star}$}

\author{
J. Hagelberg ${ }^{1,2}$, N. Engler ${ }^{1}$, C. Fontanive ${ }^{3}$, S. Daemgen ${ }^{1}$, S. P. Quanz ${ }^{1}$, J. Kühn ${ }^{1,3}$, M. Reggiani ${ }^{4}$, M. Meyer ${ }^{5}$, \\ R. Jayawardhana ${ }^{6}$, and V. Kostov ${ }^{7}$
}

\author{
${ }^{1}$ Institute for Particle Physics and Astrophysics, ETH Zurich, Wolfgang-Pauli-Strasse 27, 8093 Zurich, Switzerland \\ e-mail: janis.hagelberg@unige.ch \\ ${ }^{2}$ Geneva Observatory, University of Geneva, Chemin des Mailettes 51, 1290 Versoix, Switzerland \\ ${ }^{3}$ Center for Space and Habitability, University of Bern, Bern, Switzerland \\ ${ }^{4}$ Institute of astrophysics, KU Leuven, Celestijnlaan 200D, 3001 Leuven, Belgium \\ ${ }^{5}$ University of Michigan, Astronomy Department, USA \\ ${ }^{6}$ Department of Astronomy, Cornell University, Ithaca, NY 14853, USA \\ ${ }^{7}$ NASA/GSFC, USA
}

Received 13 August 2020 / Accepted 18 September 2020

\begin{abstract}
Context. Recent surveys indicate that planets in binary systems are more abundant than previously thought, which is in agreement with theoretical work on disc dynamics and planet formation in binaries. So far, most observational surveys, however, have focused on short-period planets in binaries, thus little is known about the occurrence rates of planets on longer periods $(\geq 10 \mathrm{au})$.

Aims. In order to measure the abundance and physical characteristics of wide-orbit giant exoplanets in binary systems, we have designed the "VIsual Binary Exoplanet survey with Sphere" (VIBES) to search for planets in visual binaries. It uses the SPHERE instrument at VLT to search for planets in 23 visual binary and four visual triple systems with ages of $<145$ Myr and distances of $<150 \mathrm{pc}$.

Methods. We used the IRDIS dual-band imager on SPHERE to acquire high-contrast images of the sample targets. For each binary, the two components were observed at the same time with a coronagraph masking only the primary star. For the triple star, the tight components were treated as a single star for data reduction. This enabled us to effectively search for companions around 50 individual stars in binaries and four binaries in triples.

Results. We derived upper limits of $<13.7 \%$ for the frequency of sub-stellar companions around primaries in visual binaries, $<26.5 \%$ for the fraction of sub-stellar companions around secondaries in visual binaries, and an occurrence rate of $<9.0 \%$ for giant planets and brown dwarfs around either component of visual binaries. We have combined our observations with literature measurements to astrometrically confirm, for the first time, that 20 binaries and two triple systems, which were previously known, are indeed physically bound. Finally, we discovered a third component of the binary HD 121336.

Conclusions. The upper limits we derived are compatible with planet formation through the core accretion and the gravitational instability processes in binaries. These limits are also in line with limits found for single star and circumbinary planet search surveys.
\end{abstract}

Key words. planets and satellites: detection - planets and satellites: dynamical evolution and stability - binaries: visual planet-star interactions

\section{Introduction}

One key statistical outcome from the more than 4000 planets that have been detected so far is that almost every Sun-like star harbours a planet (Fressin et al. 2013; Udry \& Santos 2007; Dressing \& Charbonneau 2013; Howard et al. 2012). Given that almost half of all stars in our Milky Way are bound in multiple systems (Raghavan et al. 2010), one could expect a large fraction of the detected exoplanets to be in binaries. Yet, less than 200 planets are known to reside in multiple stellar systems ${ }^{1}$ (Schwarz et al. 2016), either as circumstellar planets orbiting one of the two stars

\footnotetext{
${ }^{\star}$ Based on observations collected at the European Southern Observatory, Chile (ESO Open Time 096.C-0835, 097.C-0826, 098.C-0643, 0100.C-0543, 0101.C-0405).

1 http://www . univie.ac.at/adg/schwarz/multiple.html
}

in the binary or as circumbinary planets orbiting the centre of mass of both stars (also known as S- and P-planets, respectively, Dvorak (1982, 1984)).

Early theoretical work predicted a lower occurrence rate of circumstellar planets compared to single star planets, due to the fact that the gravitational potential of a multi-star system and truncated protoplanetary discs would hinder planet formation (Artymowicz \& Lubow 1994) and also render the planet dynamically unstable for long-term survival (Holman \& Wiegert 1999). Large exoplanet search surveys thus often avoided or gave low priority to binary stars in order to enhance the planet detection yield. Furthermore, radial velocity, transit, and direct imaging techniques all tend to have lower detection sensitivities for most binary configurations, thus further enhancing the observational bias against planets in binaries. 
Various multiplicity surveys, mostly searching with imaging for stellar companions to known radial velocity (RV) or transit detected planet hosting stars, have shown that the occurrence rate of circumstellar planets is in the $\approx 10-30 \%$ range (e.g. review of various surveys in Wang et al. 2014). However for very close binaries, which have a separation smaller than 47 au, planet occurrence is only 0.34 times the one of single stars or wider binaries (Kraus et al. 2016). For very close binaries tighter than $20 \mathrm{au}$, disc truncation and the high velocities of gas and dust induced by the secondary star render planet formation almost impossible (Zsom et al. 2011). The existence of a handful of such planets, such as $\gamma$ Cephei Ab (Hatzes et al. 2003), HD $196885 \mathrm{Ab}$ (Correia et al. 2008; Chauvin et al. 2011), and Kepler $420 \mathrm{Ab}$ (Santerne et al. 2014), may actually be the results of stellar scattering (Martí \& Beaugé 2012) instead of having formed within the binary.

In contrast to the earlier theoretical studies mentioned above, recent theoretical and observational results show that there is a possibility that planet formation in multiple stars is in fact enhanced compared to single stars as long as the binary separation is larger than 50 au. This enhancement can be caused by the secondary inducing spiral density waves in the protoplanetary disc, which can potentially stimulate gravitational instability (GI; Batygin et al. 2011; Rafikov 2013). This is in line with observations that suggest a 3-fold increase in hot Jupiter occurrence in binary stars compared to singles (Wang et al. 2015; Ngo et al. 2016; Evans et al. 2018; Fontanive et al. 2019). Binary stars should thus be taken into consideration when analysing planet formation and evolution, not only because binary stars represent $\sim 1 / 3$ of stars in the Milky Way (M: $26.8 \pm 1.4 \%$ Winters et al. 2019, FGK: $33 \pm 2 \%$ Raghavan et al. 2010, A: 32.1 ${ }_{-3.5}^{+3.9} \%$ De Rosa et al. 2014), but also to understand the robustness and diversity of planet formation.

In order to probe the population of wide companions to binary stars we started the "VIsual Binary Exoplanet survey with Sphere" (VIBES) which combines SPHERE's planet discovery and characterisation potential with its ability to simultaneously target all components of a stellar multiple, at the cost of a degraded sensitivity around the secondary star. Our survey searching for wide circumstellar planets also fills the gap between the SHINE survey targeting planets orbiting single stars and the "Search for Planets Orbiting Two Stars" (SPOTS, Thalmann et al. 2014) survey which looks for wide circumbinary planets. By combining the result on S- and P-type planet populations probed by the VIBES and SPOTS surveys respectively, we have a census of the overall population of wide giant planets in binaries. Given that a majority of our targets are in the Scorpius-Centaurus association we are probing younger but farther stars than other surveys like the NACO-LP (Chauvin et al. 2015; Desidera et al. 2015), the IDPS (Vigan et al. 2012), and the NICI Campaign (Liu et al. 2010; Biller et al. 2013) which were more sensitive to higher-mass planets at smaller orbital separations than our survey. The smaller inner working angle achieved with SPHERE and the fact that we are probing a population at wider separation mitigates the effect of larger distance.

The survey is described in the following sections starting with the target sample definition (Sect. 2). The strategy adopted for the observations and data reduction are described in Sects. 3 and 4 respectively. The confirmation of 26 binaries is described in Sect. 5, followed by the statistical analysis of the complete survey in Sect. 6, and a discussion of the survey outcome in Sect. 7.
Table 1. Astrometric and photometric uncertainties for this work and for measurements sourced in the literature.

\begin{tabular}{cccc}
\hline \hline Ref. & $\begin{array}{c}\text { Error sep. } \\
\left({ }^{\prime \prime}\right)\end{array}$ & $\begin{array}{c}\text { Error PA } \\
\left({ }^{\circ}\right)\end{array}$ & Mag. \\
\hline This work & 0.005 & 0.3 & 0.5 \\
Chauvin et al. (2003) & 0.03 & 2.5 & 0.16 \\
Daemgen et al. (2015) & 0.001 & 0.1 & 0.01 \\
Elliott et al. (2015) & 0.01 & 0.29 & 0.03 \\
Fabricius et al. (2002) & 0.14 & 1 & - \\
Gaia Collaboration (2018) & 0.0001 & 0.07 & - \\
Hartkopf et al. (1996) & 0.003 & 0.2 & - \\
Herschel et al. (1874) & - & - & - \\
Janson et al. (2013) & 0.008 & 0.4 & 0.2 \\
Jayawardhana \& Brandeker (2001) & 0.009 & 0.7 & 0.032 \\
Kouwenhoven et al. (2005) & 0.0015 & 0.03 & - \\
Kouwenhoven (2006) & 0.0015 & 0.03 & 0.12 \\
Kohler \& Leinert (1998) & 0.003 & 0.4 & - \\
McAlister et al. (1990) & 0.003 & 0.2 & - \\
Tokovinin (1997) & - & - & - \\
\hline
\end{tabular}

\section{Target sample}

We compiled a sample of 26 multiple systems that are members of young clusters and associations based on the catalogues listed in Table 1. We set selection criteria on age and distance to be younger than $50 \mathrm{Myr}$ and closer than $150 \mathrm{pc}$. Considered regions were Taurus (1-2 Myr, $d \simeq 145$ pc, Torres et al. 2009; Kraus \& Hillenbrand 2009), Scorpius Centaurus (5-15 Myr, $d \simeq 140 \mathrm{pc}$, de Zeeuw et al. 1999; Song et al. 2012; Pecaut et al. 2012), the $\beta$ Pic moving group ( $22 \pm 6 \mathrm{Myr}, d \simeq 30 \mathrm{pc}$, Binks \& Jeffries 2014; Bell et al. 2015; Shkolnik et al. 2017), Tucana-Horologium ( $45 \pm 4 \mathrm{Myr}, d \simeq 46 \mathrm{pc}$, Bell et al. 2015), and Columba (42 $2_{-4}^{+6} \mathrm{Myr}$, $d \simeq 50 \mathrm{pc}$, Bell et al. 2015). The heterogeneity induced by the wide range of age and distance in the associations has less impact on the survey sensitivity than the large variation in observing conditions, also because the final sample is dominated by ScoCen stars. Nonetheless this heterogeneity is taken into account in the final statistical analysis.

Our targets were selected in order to be bright enough ( $R<11 \mathrm{mag}$ ) to provide good adaptive optics correction, with a binary separation in the $0.8^{\prime \prime}-5^{\prime \prime}$ range to prevent $\mathrm{AO}$ wavefront sensing instabilities while still having both stars simultaneously in the IRDIS field of view. The targets' primary spectral types range between B6 and M0 with a median mass of $2.1 M_{\odot}$. The selected projected binary separations translate to $\sim 30-600$ au at their respective distances. Considering that dynamical interaction limits the maximum orbital separation of potential planetary companions on S-type orbits to $\sim 0.3$ times the binary separation on average, we should expect these planetary companions to be in the $\sim 10-170$ au range (Holman \& Wiegert 1999). The age range of $1-145 \mathrm{Myr}$ is chosen in order to optimise our planet detection sensitivity as planets are brightest when they are young (independent of the exact formation process, e.g. hotor cold-start; Fortney et al. 2008; Marley et al. 2007).

The publications out of which our sample was sourced are listed in Table 1 along with the uncertainties on the measured separation, position angle, and magnitude differences given by the authors. To determine the age of our targets we used the BANYAN $\Sigma$ tool (Gagné et al. 2018). This Bayesian analysis algorithm uses galactic coordinates (XYZ) and space velocities (UVW) of the star to compute the membership probability to 
Table 2. Associations and the age adopted for its members.

\begin{tabular}{cccc}
\hline \hline Acronym & Name & Age & Ref. \\
\hline ABD & AB Doradus & $145_{-19}^{+51}$ & Bell et al. (2015) \\
BPMG & $\beta$ Pictoris MG & $22 \pm 6$ & Shkolnik et al. (2017) \\
LCC & Lower Centaurus Crux & $17 \pm 1$ & Pecaut et al. (2012) \\
ROPH & $\rho$ Ophiuchi & $2-5$ & Wilking et al. (2008) \\
TAU & Taurus Molecular Cloud & $1-2$ & Kenyon \& Hartmann (1995) \\
THA & Tucana-Horologium Association & $45 \pm 4$ & Bell et al. (2015) \\
UCL & Upper Centaurus Lupus & $16 \pm 1$ & Pecaut et al. (2012) \\
USCO & Upper Scorpius & $11 \pm 3$ & Pecaut et al. (2012) \\
\hline
\end{tabular}

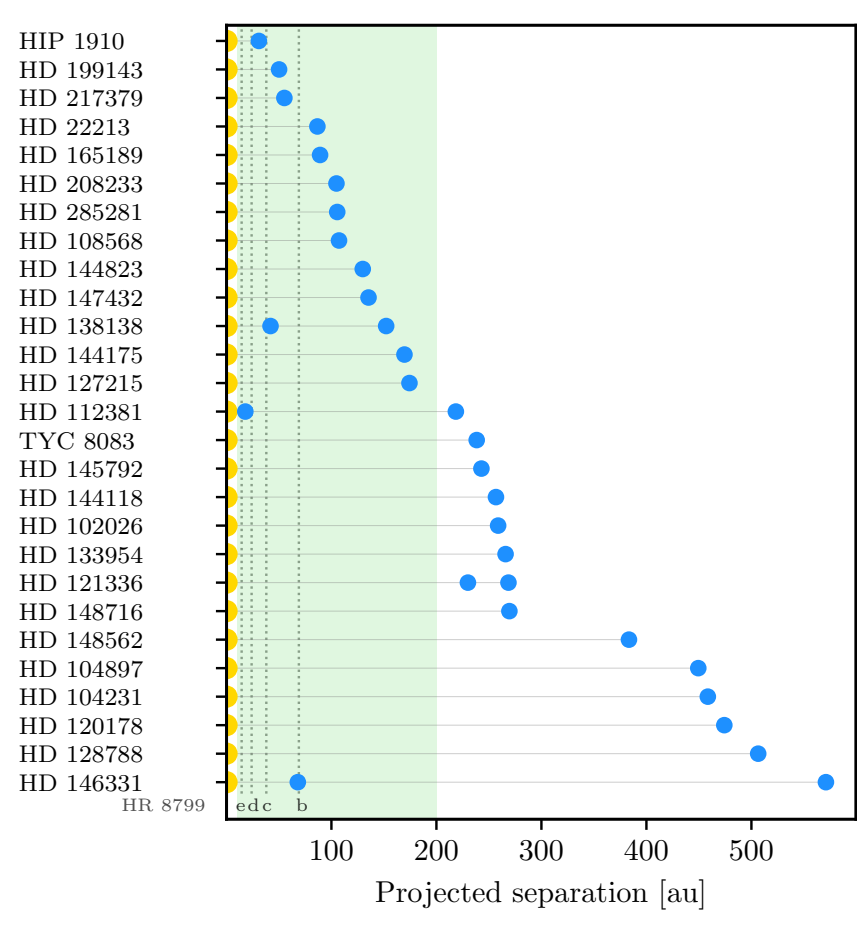

Fig. 1. Projected separation of the binaries in the observing sample. The primary and secondary stars are represented by the yellow and blue dots respectively. The green area represents the 10-200 au considered by the statistical analysis. HR 8799 bcde are represented by vertical dotted lines to illustrate the position of this wide giant planet system.

nearby young associations listed in Table 2. The results of this analysis are presented in Table A.1 giving the identified moving group to which the star is most probably associated and the membership probability. The table also lists the spectral types, coordinates, and distance given by Gaia (Gaia Collaboration 2018). Finally, for HD 102026 which was identified as a field star we used the age determined photometrically by Tetzlaff et al. (2011) of $15.8 \pm 7.3 \mathrm{Myr}$.

The sample we assembled based on these criteria is listed in Table A.1 including the existing astrometric measurements of the binaries. The spread in binary separations is illustrated in Fig. 1. It should be noted that for the triple systems HD 112381, HD 121336, HD 138138, and HD 146331 we are not sensitive to planets which would be orbiting in between the two tight components of these hierarchical triple systems. Except for HD 217379 and HD 285281 that have an estimated age of $\sim 145 \mathrm{Myr}$ and $\sim 45 \mathrm{Myr}$ respectively, all remaining targets in the sample have an age less than $22 \mathrm{Myr}$ as illustrated in Fig. 2.

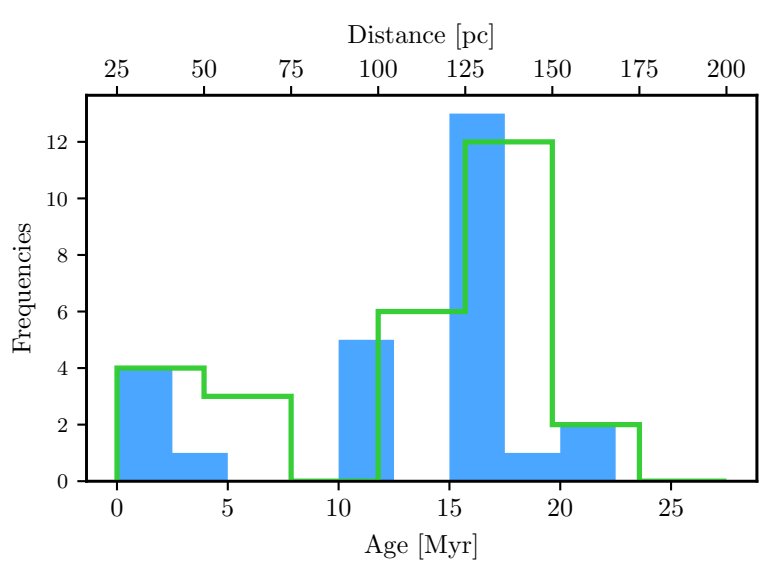

Fig. 2. Histogram of age and distance of the VIBES sample, blue bars and green line respectively. HD 217379 and HD 285281 (145 and $45 \mathrm{Myr}$ respectively) have been left out in the age histogram for better readability.

\section{Observational strategy}

The observations were carried out with SPHERE at VLT (Beuzit et al. 2008) in IRDIFS mode which simultaneously acquires data in dual-band imaging through the $\mathrm{H} 2 \mathrm{H3}$ filters $\left(\lambda_{H 2}=\right.$ $1.593 \pm 0.055 \mu \mathrm{m} ; \lambda_{H 3}=1.667 \pm 0.056 \mu \mathrm{m}$, Vigan et al. 2010) with IRDIS (Dohlen et al. 2008), and integral field spectroscopy in $Y-J\left(0.95-1.35 \mu \mathrm{m}, R_{\lambda} \sim 54\right)$ with the IFS (Claudi et al. 2008). Both instruments are situated behind the 185 mas diameter apodized Lyot coronagraph (Carbillet et al. 2011; Guerri et al. 2011) which is masking the brighter star in $H$ band. The observations were all carried out in pupil-tracking (i.e. where the field of view rotates), in order to apply Angular Differential Imaging (ADI, Schneider \& Silverstone 2003; Liu 2004; Marois et al. 2006) as described in Sect. 4.

The same observing sequence is carried out for each target, which starts with calibrations followed by science observations. The calibration sub-sequence is composed of long exposure sky frames taken by offsetting the stars out of the field of view in order to carry out bad pixel correction and to estimate the background flux. We rely on unsaturated non-coronagraphic exposures with both stars in the field-of-view to calibrate the photometry, the point spread function (PSF) profile, and the astrometric configuration of the targeted binary system. The last part of the calibration sequence are the star centring frames where satellite spots are generated through a sinusoidal pattern on the deformable mirror (Jovanovic et al. 2015; Rickman et al. 2020) in order to determine precisely the position of the primary star behind the coronagraph. Typically the total observing time 
dedicated to each target is an hour, which besides the $15 \mathrm{~min}$ spent on telescope slewing and target acquisition is split in $80 / 20 \%$ between the science observation and calibrations.

A certain number of targets have been carried out through an ESO filler programme, which implies bad weather conditions and sub-optimal field rotation. In certain cases these targets have been re-observed in better conditions and with larger field rotation (Table A.2).

The observational strategy exploits the large field of view of IRDIS to simultaneously acquire both stars of all selected binaries. In principle this doubles the total number of stars probed in comparison to a single star survey. However the sensitivity around the second star is lower because the star is not behind a coronagraph, and the field of view gets smaller as the secondary star gets closer to the edge of the detector.

Second epoch follow-up observations were carried out on all sub-stellar candidates in order to verify if they are co-moving and thus physically bound. For these observations the star centring satellite spots were kept during the whole observing sequence to improve the astrometric fitting in case of a confirmed planetary companion detection.

\section{Data reduction}

The IRDIS data reduction is based on GRAPHIC (Hagelberg et al. 2016), with modifications in order to have an end-to-end reduction for SPHERE data (Cheetham et al. 2018). The pipeline cosmetics pre-processing involves sky subtraction, flat fielding, bad pixel correction, filter splitting, distortion correction (based on Maire et al. (2016)) and individual frame registration. For the PSF subtraction we use ADI combined with algorithms based on principal component analysis (PCA; Amara \& Quanz 2012; Soummer et al. 2012) applied on $2 \times F W H M$ wide concentric annular sections. In order to minimise companion self-subtraction we exclude frames where the field rotation is less than $1.25 \times F W H M$ (the detailed procedure is described in Cheetham et al. (2018)). The final images are then derotated in Fourier space and median combined to produce the final image.

Additionally a Spectral Differential Imaging (SDI, Sparks \& Ford 2002) reduction is also used where the $H 3$ filter image is spatially and flux rescaled by the wavelength ratio and subtracted from the simultaneous $H 2$ image. The resulting frames are then run through the same PCA algorithm. This Angular and Spectral Differential Imaging (ASDI) data reduction product thus adds a third potential companion detection image to the two ADI reductions of the $H 2$ and $H 3$ filters.

The IFS data reduction uses the SPHERE Data Reduction and Handling pipeline (DRH, Pavlov et al. 2008) to produce calibrated data. This calibration includes the background subtraction, bad pixel correction, the wavelength calibration, correction for the spectral cross-talk (Mesa et al. 2015) and the flat fielding. The wavelength-dependent centring of the frames is performed using the satellite spots. The extracted IFS data cube consists of temporal sequences of 39 monochromatic images with a format of $290 \times 290$ pixels each, which are then processed using the PCA-based pipeline PynPoint (Stolker et al. 2019).

Companions close to the secondary star may not be uncovered with a data reduction centred on the primary star because of the typical azimuthal smearing of bright field components from the ADI processing. More importantly ADI (and ASDI) rely on the fact that the target PSF is not rotating, the data reduction can thus only be optimised for one central PSF at a time. The only changes in the data reduction for the secondary component is that the centring is done by fitting a 2D Gaussian to the secondary

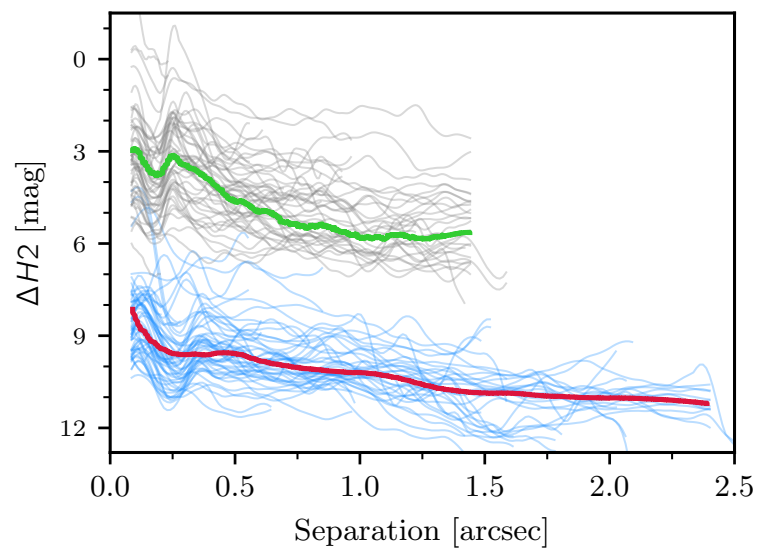

Fig. 3. All IRDIS H2 contrast curves for the primary and secondary stars, blue and grey respectively, along with their median contrast curves in red and green respectively.

PSF instead of using the satellite spots. This comes from the fact that the secondary component is not behind a coronagraph so that the satellite spots disappear in the stellar halo. For similar mass binaries the PSF of the secondary is slightly saturated but still usable for a Gaussian fit.

Each telescope pointing thus results in at least five data products, two IRDIS reductions for each star in the field and one IFS reduction for the brightest star in the field. In the case of triple systems we only reduced the two brightest components as the third star was in every case too close to one of the brighter stars.

Contrast curves are then computed by first estimating the noise as a function of the separation to the central star by measuring the standard deviation in concentric $\lambda / D$ wide annuli. These contrast curves are then calibrated for throughput by injecting fake-planets based on a non-saturated PSF of the star before PCA subtraction. Following the method proposed by Mawet et al. (2014) the injection is done in order to keep a constant false positive rate of $2.9 \times 10^{-7}$ to correct for small sample statistics. The procedure is repeated 10 times with a varying azimuth. Systematic effects such as detector defects are not taken into account which thus results in a more conservative detection limit.

All the IRDIS $H 2$ contrast curves for the primary and secondary stars are given in Fig. 3 with an arbitrary cut-off at $\sim 1.5$ and $\sim 2.5$ arcseconds for readability, along with the median contrast curves of the primaries and of the secondaries. $H 3$ yielded a similar sensitivity while ASDI and IFS contrast curves where not used as they were deemed not robust enough for a statistical analysis, even though ASDI and ADI-IFS data reduction was done.

\section{Observational results}

Except for HIP 1910 (Chauvin et al. 2003), HD 165189 (Herschel et al. 1874), HD 285281 (McAlister et al. 1990; Daemgen et al. 2015) which were already identified as co-moving binaries, and HD 138138 (Hartkopf et al. 1996; Kohler \& Leinert 1998) which is a known triple system, all the other targets in our sample were suggested to be physically bound prior to our observations only based on a statistical approach showing that the probability of the secondary to be a background object was low (Chauvin et al. 2003; Daemgen et al. 2015; Elliott et al. 2015; Fabricius et al. 2002; Gaia Collaboration 2018; Hartkopf et al. 1996; Herschel et al. 1874; Janson et al. 2013; Kouwenhoven et al. 2005; Kouwenhoven 2006; Kohler \& Leinert 1998; McAlister et al. 1990; Tokovinin 1997). As all our targets have now been 


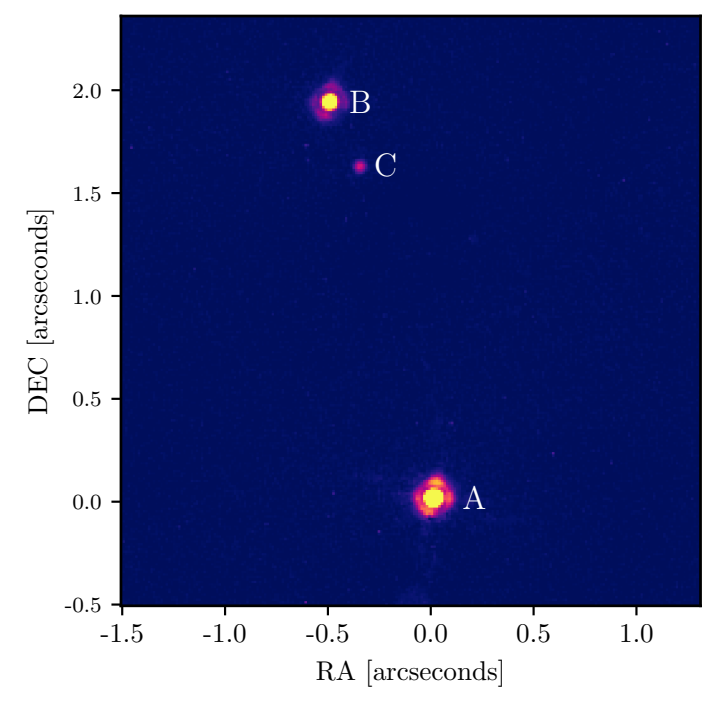

Fig. 4. Binary HD $121336 \mathrm{AB}$ along with the third newly discovered component $\mathrm{C}$.

observed at least once by our programme we are able to verify through astrometry whether the systems in our sample are indeed gravitationally bound. The more than 5 years' time baseline between the binary discovery observations and our observations is sufficient for an unambiguous determination of the binaries' physical link.

In order to measure the position angle (PA), separation, and magnitude difference between the primary and secondary star we have used the photometric calibration frames taken at the beginning of every sequence. In this data all the PSFs are noncoronagraphic and unsaturated ensuring the best photometric and astrometric accuracy. The PSF measurements are carried out using the DAOPHOT FIND algorithm (Stetson 1987) as implemented in Astropy (Astropy Collaboration 2013) where peaks over a given threshold are searched and a two-component Gaussian is then fitted to that peak. The resulting FWHM fit in $\mathrm{x}$ and $\mathrm{y}$ is used to determine the roundness of the PSF. From these measurements we determine the astrometric position as well as the brightness of the companion with respect to the primary star. Given that all observations are taken in pupil stabilised mode we need to take into account the parallactic angle correction when deriving the position angle (PA). This angle correction is calculated by GRAPHIC (Hagelberg et al. 2016). The resulting PA measurement when including PSF fitting (estimated at 0.4 pixel), true north determination (Maire et al. 2016), and instrument pupil offset results in a 0.3 degree uncertainty. The separation measurement once corrected for the anamorphic distortion between $\mathrm{x}$ and $\mathrm{y}$ axis is mostly dependent on the PSF fitting accuracy resulting in an estimated $0.005^{\prime \prime}$ uncertainty on the separation. For the magnitude measurement we have used the peak value of the Gaussian fit on the primary and companion star. The magnitude of the companion star is then derived by taking the 2MASS $H$ band magnitude (Skrutskie et al. 2006) of the primary and applying the magnitude difference. This quick first order approach is thus not taking into account the difference between the 2MASS- $H$ and IRDIS- $H 2$ filters, which thus yields an overall uncertainty of $0.5 \mathrm{mag}$ on the companion star magnitude.

A third faint stellar component was detected in the HD 121336 AB binary (Fig. 4). Given the faintness and close proximity to the secondary it probably was beyond reach of previous instruments so that only a single epoch is available for the moment. Using BT-Settl models with Solar metallicity
(Allard 2014) we estimate the mass of component $C$ to be around $0.95 M_{\odot}$. Given that component $\mathrm{C}$ is only at $0.346^{\prime \prime}$ of $\mathrm{B}$ (with a PA of $205.3 \mathrm{deg}$ and $\Delta H=2.3$ with respect to $\mathrm{B}$ ), we can assume that B-C form a binary orbiting $\mathrm{A}$, if it is bound.

Table 1 summarises the uncertainties on the measured stellar companions' PAs, separations, and magnitudes as well as those reported in the literature we used to source previous measurements. The measurements from this and previous work for the binaries are listed in Table A.3.

To determine if the secondary component is bound or not we plot the positions from all epochs (orange crosses in Fig. A.1) and compare them to the relative motion a background object would exhibit (blue lines). The line starts at the companion coordinates of the first observed position and then shows the astrometric track an object would follow with respect to the primary star if it had no proper motion. The green crosses along this line represent the positions a background object would have at the given observing dates. The wobbles are caused by the parallactic motion. The proper motion we used is based on Gaia (Gaia Collaboration 2016; Lindegren et al. 2018) and HippARCOs (Perryman et al. 1997) and it should be noted that all the parallax measurements were graded as best quality, which means that the double star nature of the targets did not interfere with the observations.

Figure A.2 show the positions of the secondary star at all epochs. The primary star is represented by the blue cross at the origin of the polar plot, while the red dots represent the secondary positions. The orange error bars on the secondary positions are mostly invisible due to the precision of the measurements. A green curve is also plotted to illustrate the counter-clockwise movement the secondary would have if it was on a face-on circular orbit with the observed time baseline. The apparent movement caused by the proper motion is not included in this plot as it would be too small to be visible. Orbital motion is clearly visible in the $\sim 150$ years binary HIP 1910 AB.

From this analysis, we can astrometrically confirm that 21 previously known visible binaries and three triple systems are indeed physically bound. The only exception are HD 165189 which was already known to be bound and HD 121336 AB where the binary is proven to be bound for the first time through astrometry but where a third component is also detected for the first time.

\section{Statistical analysis}

We are able to place upper limits on the occurrence rates of brown dwarfs and giant planets in binary systems. We must also rely on model predictions for the underlying population distributions in order to constrain these frequencies. As wideorbit exoplanets and brown dwarfs in stellar binaries have yet to be studied in depth on the theoretical side, we use population synthesis models developed for single stars, and assume that circumprimary sub-stellar companions follow similar distributions in mass and separation. Our model population consists of two sub-populations, based on the GI (Boss 1998) and core accretion (CA, Mizuno 1980; Lin \& Papaloizou 1986; Pollack et al. 1996) formation scenarios. The GI synthetic population comes from the disc fragmentation simulations first presented in Forgan \& Rice (2013) and later updated by Forgan et al. (2018). The synthetic CA population was obtained from the latest version of the Bern models of planetary formation and evolution (Mordasini 2018), and corresponds to population NG76 from the new generation planetary population synthesis (NGPPS) series 
(Emsenhuber et al. 2020a,b). Combined together, these two population synthesis models form the full population model used in our statistical analysis. The CA formation channel predicts lower-mass and closer-separation planets, while the GI mechanism predicts more massive brown dwarfs on wider orbits (see e.g. Vigan et al. 2020). The CA and GI populations serve as a first order approach as they are based on single star models which do not take into account an outer perturber. CA populations should be affected by truncation of the disc (Artymowicz \& Lubow 1994), an increase of the random velocities of the planetesimals, and a possible destabilisation of the planetary system through $\mathrm{N}$ body interactions with the binary, including Kozai (Lidov 1962; Kozai 1962). The outer stellar perturber could stabilise the protoplanetary disc which would inhibit GI planet formation (Forgan \& Rice 2009) but it could also trigger spiral density waves which would spark GI (Batygin et al. 2011; Rafikov 2013).

In order to constrain the statistical properties of our observed sample, we first converted the obtained detection limits for each target into detection probability maps in terms of companion masses and semi-major axes. We used the COND-2003 evolutionary tracks (Baraffe et al. 2003) to convert observed contrasts into companion mass using the respective magnitude, distance and age of each target. Given the large range in the levels of contrast obtained, we found these models to be the only ones covering the full span of achieved detection limits. We then followed the Monte-Carlo procedure described in Vigan et al. (2020) to perform the de-projection of the limits from projected separation onto semi-major axis. We adopted for this the Beta distribution from Bowler et al. (2020) to describe the eccentricity distribution of the overall population of wide sub-stellar companions.

The resulting survey completeness is presented in Fig. A.3, showing the average two-dimensional completeness around the stellar primaries (left panel), stellar secondaries (middle panel) and considering all binary components together (right panel). These provide the average probability of detecting an object of given mass and semi-major axis. The poorer contrasts achieved around the secondaries result in a significant loss in the depth of the mass limits reached around these stars compared to the primaries. In addition, some detection limits were cut at very short projected separations due to the small angular separation of the stellar binary system. As the limits around the most distant stars in the sample start at wider physical separations than the cuts made on some of these targets, there is no region in the parameter space with a completeness fraction of 1 .

From the derived completeness maps and adopted model populations, we constrained the frequencies of sub-stellar companions in visual stellar binaries using the statistical code from Fontanive et al. (2018). This Markov Chain Monte Carlo (MCMC) sampling tool was built using the EMCEE (ForemanMackey et al. 2013) python algorithm, and aims at placing statistical constraints on stellar and sub-stellar populations (occurrence rates, mass and separation distributions of companions) based on observed surveys (e.g. Fontanive et al. 2018, 2019; Vigan et al. 2020). We performed separately the same analyses on the 27 stellar primaries, the 27 secondaries, and the combined 54 binary components, in order to place constraints on the occurrence rates of sub-stellar companions around primaries, secondaries and either component of wide binary systems, respectively. In all cases, we fitted the relative frequencies associated with each part of the model population and derive the total fraction of sub-stellar companions as the sum of the two parts, as was done in Vigan et al. (2020).

Based on the average completeness maps presented in Fig. A.3, we chose to focus on the region of parameter space ranging from 10 to $200 \mathrm{au}$ in semi-major axis, and from 10 to $75 M_{\text {Jup }}$ in mass. We used uniform priors between 0 and 1 for the two companion fractions. In each simulation, the MCMC code was run with 2000 walkers taking 5000 steps each. We found that convergence of the chains was reached after a couple hundred steps and discarded the initial 500 steps as the "burn-in" phase. The results from the three analyses performed are presented in Fig. A.4, for the primaries (left), secondaries (centre) and all stars (right). The posterior distributions of the companion fractions associated with the GI and CA formation models are shown in red and blue, respectively. The yellow curves are inferred from the sum of the GI and CA frequencies at each step in the MCMC, and thus provide the total fraction of sub-stellar companions between 10-200 au and 10-75 $M_{\text {Jup }}$.

We obtained upper limits for all cases. In general, roughly comparable limits were placed on both parts of the model, since the lack of detected companions does not allow us to scale the relative fractions between the GI and CA populations. The posteriors of the CA part (blue) are slightly wider due to the lower completeness levels achieved in the regions of the parameter space where most CA planets are predicted. This effect is emphasised for the secondaries (middle panel) around which the completeness reached is strongly decreased at the lowest masses and separations. The much lower completeness fraction reached around the secondaries in the probed parameter space (see Fig. A.3) is also responsible for the overall looser constraints derived for this subset. The shaded yellow areas in Fig. A.4 indicate the $68 \%$ confidence intervals for the full sub-stellar companion occurrences rates. The resulting values (1- $\sigma$ level) are: $<13.7 \%$ for the frequency of sub-stellar companions around primaries in visual binaries, $<26.5 \%$ for the fraction of sub-stellar companions around secondaries in visual binaries, and an occurrence rate of $<9.0 \%$ for giant planets and brown dwarfs around either component of visual binaries. Sample size is clearly a driving factor in the obtained results, with the tightest constraints derived the full sample of 54 stars. Of course, these results are only valid under the assumption that the GI and CA theories are the only possible formation channels for brown dwarfs and giant planets in the explored mass and separation ranges.

\section{Discussion}

Vigan et al. (2020) recently presented the first statistical results from the SPHERE GTO F150 (Desidera et al. 2020) single star sub-sample from the SHINE (SpHere INfrared survey for Exoplanets, Chauvin et al. 2017) survey. This sub-sample is based on the first half of SHINE targets observed excluding bad condition observations and newly discovered stellar binaries. Our results appear to be broadly consistent with the frequencies of sub-stellar companions reported in Vigan et al. (2020) for single stars, although a direct comparison between the VIBES and SHINE F150 sample is not trivial. Indeed, our targets cover a wide range of spectral types, while the statistical analyses performed in Vigan et al. (2020) considered three different spectral bins, with different associated population models. The most relevant comparison to our results is arguably the constraints they obtained for FGK stars using the same GI and CA synthetic populations as in the present work, for which they derived a companion fraction of $5.7_{-2.8}^{+3.8} \%$ between $1-75 M_{\text {Jup }}$ and 5$300 \mathrm{au}$. This is in agreement with our obtained value of $<9 \%$ for our full sample of 54 stars over the ranges 10-75 $M_{\text {Jup }}$ and 10-200 au, noting that this fraction would be enhanced over the wider parameter space probed in the SHINE survey. Since our sample of secondary stars is somewhat biased towards lower 
stellar masses, we also compare the results from our analysis on secondaries to the occurrence rate measured by Vigan et al. (2020) for $M$ dwarfs. Our loose constraints of $<26.5 \%$ is also consistent with the frequency of $12.6_{-7.1}^{+12.9} \%$ and $4.7_{-1.5}^{+2.0} \%$ from the SHINE (Vigan et al. 2020) and GPIES (5-80 M Jup, 10-100 au, Nielsen et al. 2019) surveys respectively. Although we note again that the two analyses are not directly comparable and that slightly different companion mass and semi-major axis ranges were explored.

Several studies have suggested that systems with giant planets on very tight orbits may be more likely to host a wide stellar companion (Eggenberger et al. 2004; Desidera \& Barbieri 2007; Mugrauer et al. 2007). In the Friends of hot Jupiters campaign, Ngo et al. (2016) found that the occurrence of 50-2000 au binary companions is strongly increased when hot Jupiters are present, and concluded that binarity plays a role in the formation or evolution of such giant planets. More recently, Fontanive et al. (2019) searched for stellar companions to stars hosting planets and brown dwarfs companions with masses $>7 M_{\text {Jup }}$ within 1 au and measured a further inflated binary fraction of $\sim 80 \%$ on separations in the range $20-10000$ au. These results confirmed the trends observed for lower-mass planets, and suggested that the effects of stellar multiplicity on the presence of massive close-in companions may be enhanced for higher-mass planets and brown dwarfs. The study conducted by Fontanive et al. (2019) is of particular relevant for imaging programmes as the masses of the inner companions probed in that survey (7-60 $M_{\text {Jup }}$ ) are comparable to the typical masses of sub-stellar companions detectable with direct imaging. This mass range is indeed very similar to the mass interval explored in the statistical analyses presented in Sect. 6 (10-75 $\left.M_{\mathrm{Jup}}\right)$.

It has been proposed that these massive planets and brown dwarfs detected on short periods originally formed at wider orbital separations (i.e. where the directly-imaged population lies) and that their inward migration was induced or facilitated by the gravitational influence of a stellar companion ( $\mathrm{Ngo}$ et al. 2016; Fontanive et al. 2019). Although the precise stages in the system's evolution at which these processes would take place are not clear, we would expect in this case a number of giant planets and brown dwarf companions to reside at large separations in extremely young binary star systems. This makes targets like the ones probed in the VIBES survey potentially promising samples for the direct detection of sub-stellar companions.

However, even if binarity is indeed required to obtain massive planets on hot Jupiter orbits, such systems remain sparse and this scenario must hence be a rather rare event (there are less than 40 known systems with masses $>7 M_{\text {Jup }}$ within 1 au despite the magnified sensitivity to bigger and more massive companions with the transit and radial velocity detection methods; see Fontanive et al. 2019). The low occurrence of such a mechanism could be due to the migration processes involved being somewhat inefficient, or be a direct consequence of low formation rates of wide giant planets in binaries. Therefore, the positive correlation between the existence of massive short-period planets and stellar multiplicity may not necessarily imply a higher frequency of massive planets in binaries. The null detection from our survey appears to confirm this idea, although larger sample sizes will be needed to more robustly measure the frequency of distant sub-stellar companions among visual binaries compared to single stars.

The SPOTS survey (Thalmann et al. 2014; Bonavita et al. 2016; Asensio-Torres et al. 2018) which searched for companions in the $\sim 30-300$ au range around tight binaries found upper limits on the frequency of circumbinary planets (1-15 $\left.M_{\mathrm{Jup}}\right)$ and brown dwarfs (16-70 $\left.M_{\text {Jup }}\right)$ of 10 and $6 \%$ respectively, at a $95 \%$ confidence level. Contrary to VIBES, the SPOTS survey sensitivity was only marginally impacted by the binary nature of the targets yielding tighter upper limits but still no planet detection. Circumbinary discs are thought not to be perturbed by the binary star beyond two to four times the binary separation (Holman \& Wiegert 1999) so that the formation history would be very different from the intra-binary planet formation where the secondary star acts as an external perturber on the disc and on the planet orbit (Ngo et al. 2016; Fontanive et al. 2019). The results of the SPOTS survey even though probing a different type of binary planet population is compatible with our results.

\section{Conclusion}

We presented the VIBES. We observed a total of 23 binaries and 4 triple systems with SPHERE in IRDIFS mode. The main results of the survey are as follows:

- We have searched for sub-stellar companions around 46 stars which were part of a binary system, around 4 stars in triples which were orbiting another binary star, and around 4 binaries in triples which were orbiting another star. No sub-stellar companion was detected as all companion candidates were followed up and turned out to be background objects;

- We derived $(1-\sigma$ level) upper limits of $<13.7 \%$ for the frequency of sub-stellar companions around primaries in visual binaries, $<26.5 \%$ for the fraction of sub-stellar companions around secondaries in visual binaries, and an occurrence rate of $<9.0 \%$ for giant planets and brown dwarfs (10-75 $\left.M_{\text {Jup }}\right)$ around either component of visual binaries, in the 10-200 au range;

- We confirmed 20 binaries and 2 triple systems to be comoving and thus physically bound;

- One new $0.95 M_{\odot}$ star was detected in the previously known binary system HD 121336;

The upper limits we have derived for planets in binaries are compatible with frequencies obtained in single stars direct imaging surveys such as SHINE $\left(12.6_{-7.1}^{+12.9} \%\right.$, Vigan et al. 2020) and GPIES (4.7 $7_{-1.5}^{+2.0} \%$, Nielsen et al. 2019). A direct comparison is however not possible given the difference in parameter ranges probed by these three surveys.

Studies of planets in binaries has increased in recent years specially through the search of stellar companions to transiting and RV planets, revealing the short period planets population. To probe the wider orbit planets direct imaging remains the primary technique, however current direct imaging techniques have a limited performance in the search of planets within close binaries. Dual star coronagraphs (Cady et al. 2011; Aleksanyan et al. 2017; Kühn et al. 2018) and multi-star wavefront control techniques (Sirbu et al. 2017) offer promising prospects to significantly increase planet sensitivity in binaries. Nonetheless statistical completeness is limited by the varying separation range which is set by the binary separation instead of the field of view.

Acknowledgements. We would like to thank Markus Janson for his input on the binaries he has observed, Ken Rice and Christoph Mordasini for useful discussions on population models, and the anonymous referee for the very constructive comments which significantly improved the scientific quality of the article. J.H. is supported by SNSF through the Ambizione grant \#PZ00P2_180098. J.H., N.E., S.Q., J.K. acknowledge the financial support from the SNSF through the National Centre for Competence in Research "PlanetS". This research has made use of the SIMBAD database, operated at CDS, Strasbourg, France, and of NASA's Astrophysics Data System. This research made use of Astropy, a community-developed core Python package for Astronomy (Astropy Collaboration 2013), as well as numpy (Walt et al. 2011), and pandas (Mckinney 2011). 


\section{References}

Aleksanyan, A., Kravets, N., \& Brasselet, E. 2017, Phys. Rev. Lett., 118, 203902 Allard, F. 2014, in Proc. IAU, 299, 271

Amara, A., \& Quanz, S. P. 2012, MNRAS, 427, 948

Artymowicz, P., \& Lubow, S. H. 1994, ApJ, 421, 651

Asensio-Torres, R., Janson, M., Bonavita, M., et al. 2018, A\&A, 619, A43

Astropy Collaboration (Robitaille, T. P., et al.) 2013, A\&A, 558, 33

Baraffe, I., Chabrier, G., Barman, T. S., Allard, F., \& Hauschildt, P. H. 2003, A\&A, 402, 701

Batygin, K., Morbidelli, A., \& Tsiganis, K. 2011, A\&A, 533, A7

Bell, C. P. M., Mamajek, E. E., \& Naylor, T. 2015, MNRAS, 454, 593

Beuzit, J.-L., Feldt, M., Dohlen, K., et al. 2008, Proc. SPIE, 7014, 476

Biller, B. A., Liu, M. C., Wahhaj, Z., et al. 2013, ApJ, 777, 160

Binks, A. S., \& Jeffries, R. D. 2014, MNRAS, 438, L11

Bonavita, M., Desidera, S., Thalmann, C., et al. 2016, A\&A, 593, A38

Boss, A. P. 1998, ApJ, 503, 923

Bowler, B. P., Blunt, S. C., \& Nielsen, E. L. 2020, AJ, 159, 63

Cady, E., McElwain, M., Kasdin, N. J., \& Thalmann, C. 2011, Publ. Astron. Soc. Pac., 123, 333

Carbillet, M., Bendjoya, P., Abe, L., et al. 2011, Exp. Astron., 30, 39

Chauvin, G., Thomson, M., Dumas, C., et al. 2003, A\&A, 404, 157

Chauvin, G., Beust, H., Lagrange, A.-M., \& Eggenberger, A. 2011, A\&A, 528, A8

Chauvin, G., Vigan, A., Bonnefoy, M., et al. 2015, A\&A, 573, A127

Chauvin, G., Desidera, S., Lagrange, A.-M., et al. 2017, A\&A, 605, L9

Cheetham, A., Ségransan, D., Peretti, S., et al. 2018, A\&A, 614, A16

Claudi, R. U., Turatto, M., Gratton, R. G., et al. 2008, in Astronomical Telescopes and Instrumentation: Synergies Between Ground and Space, 70143E

Correia, A. C. M., Udry, S., Mayor, M., et al. 2008, A\&A, 479, 271

Daemgen, S., Bonavita, M., Jayawardhana, R., Lafrenière, D., \& Janson, M. 2015, ApJ, 799, 155

De Rosa, R. J., Patience, J., Wilson, P. A., et al. 2014, MNRAS, 437, 1216

Desidera, S., \& Barbieri, M. 2007, A\&A, 462, 345

Desidera, S., Covino, E., Messina, S., et al. 2015, A\&A, 573, A126

Desidera, S., Chauvin, G., Bonavita, M., et al. 2020, A\&A, submitted

de Zeeuw, P. T., Hoogerwerf, R., de Bruijne, J. H. J., Brown, A. G. A., \& Blaauw, A, 1999, AJ, 117, 354

Dohlen, K., Langlois, M., Saisse, M., et al. 2008, Proc. SPIE, 7014, 1266

Dressing, C. D., \& Charbonneau, D. 2013, ApJ, 767, 95

Dvorak, R. 1982, Oesterreich. Akad. Wissensch. Math. Naturwiss. Klasse Sitzungsb. Abteil., 191, 423

Dvorak, R. 1984, Celest. Mech., 34, 369

Eggenberger, A., Udry, S., \& Mayor, M. 2004, A\&A, 417, 353

Elliott, P., Huélamo, N., Bouy, H., et al. 2015, A\&A, 580, A88

Emsenhuber, A., Mordasini, C., Burn, R., et al. 2020a, A\&A, submitted [arXiv:2007.05561]

Emsenhuber, A., Mordasini, C., Burn, R., et al. 2020b, A\&A, submitted [arXiv:2007.05562]

Evans, D. F., Southworth, J., Smalley, B., et al. 2018, A\&A, 610, A20

Fabricius, C., Høg, E., Makarov, V. V., et al. 2002, A\&A, 384, 180

Fontanive, C., Biller, B., Bonavita, M., \& Allers, K. 2018, MNRAS, 479, 2702

Fontanive, C., Rice, K., Bonavita, M., et al. 2019, MNRAS, 485, 4967

Foreman-Mackey, D., Hogg, D. W., Lang, D., \& Goodman, J. 2013, Publ. Astron. Soc. Pac., 125, 306

Forgan, D., \& Rice, K. 2009, MNRAS, 400, 2022

Forgan, D., \& Rice, K. 2013, MNRAS, 432, 3168

Forgan, D. H., Hall, C., Meru, F., \& Rice, W. K. M. 2018, MNRAS, 474, 5036

Fortney, J. J., Marley, M. S., Saumon, D., \& Lodders, K. 2008, ApJ, 683, 1104

Fressin, F., Torres, G., Charbonneau, D., et al. 2013, ApJ, 766, 81

Gagné, J., Mamajek, E. E., Malo, L., et al. 2018, ApJ, 856, 23

Gaia Collaboration (Prusti, T., et al.) 2016, A\&A, 595, A1

Gaia Collaboration (Brown, A. G. A., et al.) 2018, A\&A, 616, A1

Guerri, G., Daban, J.-B., Robbe-Dubois, S., et al. 2011, Exp. Astron., 30, 59

Hagelberg, J., Ségransan, D., Udry, S., \& Wildi, F. 2016, MNRAS, 455, 2178

Hartkopf, W. I., Mason, B. D., McAlister, H. A., et al. 1996, AJ, 111, 936

Hatzes, A. P., Cochran, W. D., Endl, M., et al. 2003, ApJ, 599, 1383

Herschel, J. F. W., Main, R., \& Pritchard, C. 1874, Mem. Roy. Astron. Soc., 40, 1

Holman, M. J., \& Wiegert, P. A. 1999, AJ, 117, 621
Howard, A. W., Marcy, G. W., Bryson, S. T., et al. 2012, ApJS, 201, 15 Janson, M., Lafrenière, D., Jayawardhana, R., et al. 2013, ApJ, 773, 170 Jayawardhana, R., \& Brandeker, A. 2001, ApJ, 561, L111 Jovanovic, N., Guyon, O., Martinache, F., et al. 2015, ApJ, 813, L24

Kenyon, S. J., \& Hartmann, L. 1995, ApJS, 101, 117

Kohler, R., \& Leinert, C. 1998, A\&A, 331, 977

Kouwenhoven, M. B. N. 2006, PhD thesis, University of Amsterdam, The Netherlands

Kouwenhoven, M. B. N., Brown, A. G. A., Zinnecker, H., Kaper, L., \& Portegies Zwart S. F. 2005, A\&A, 430, 137

Kozai, Y. 1962, AJ, 67, 579

Kraus, A. L., \& Hillenbrand, L. A. 2009, ApJ, 704, 531

Kraus, A. L., Ireland, M. J., Huber, D., Mann, A. W., \& Dupuy, T. J. 2016, AJ, 152,8

Kühn, J., Daemgen, S., Wang, J., et al. 2018, Proc. SPIE, 10702, 1070242

Lidov, M. L. 1962, Planet. Space Sci., 9, 719

Lin, D. N. C., \& Papaloizou, J. 1986, ApJ, 307, 395

Lindegren, L., Hernández, J., Bombrun, A., et al. 2018, A\&A, 616, A2

Liu, M. C. 2004, Science, 305, 1442

Liu, M. C., Wahhaj, Z., Biller, B. A., et al. 2010, Proc. SPIE, 7736, 77361K

Maire, A.-L., Langlois, M., Dohlen, K., et al. 2016, Proc. SPIE, 9908, 990834

Marley, M. S., Fortney, J. J., Hubickyj, O., Bodenheimer, P., \& Lissauer, J. J. 2007, ApJ, 655, 541

Marois, C., Lafrenière, D., Doyon, R., Macintosh, B., \& Nadeau, D. 2006, ApJ, 641,556

Martí, J. G., \& Beaugé, C. 2012, A\&A, 544, A97

Mawet, D., Milli, J., Wahhaj, Z., et al. 2014, ApJ, 792, 97

McAlister, H., Hartkopf, W. I., \& Franz, O. G. 1990, AJ, 99, 965

Mckinney, W. 2011, Python High Perform. Sci. Comput.

Mesa, D., Gratton, R., Zurlo, A., et al. 2015, A\&A, 576, A121

Mizuno, H. 1980, Progr. Theoret. Phys., 64, 544

Mordasini, C. 2018, Handbook of Exoplanets, (Cham: Springer), 143

Mugrauer, M., Neuhäuser, R., \& Mazeh, T. 2007, A\&A, 469, 755

Ngo, H., Knutson, H. A., Hinkley, S., et al. 2016, ApJ, 827, 8

Nielsen, E. L., De Rosa, R. J., Macintosh, B., et al. 2019, AJ, 158, 13

Pavlov, A., Möller-Nilsson, O., Feldt, M., et al. 2008, Adv. Softw. Control Astron. II, 7019, 701939

Pecaut, M. J., Mamajek, E. E., \& Bubar, E. J. 2012, ApJ, 746, 154

Perryman, M. a. C., Lindegren, L., Kovalevsky, J., et al. 1997, A\&A, 500, 501

Pollack, J. B., Hubickyj, O., Bodenheimer, P., et al. 1996, Icarus, 124, 62

Rafikov, R. R. 2013, ApJ, 765, L8

Raghavan, D., McAlister, H. A., Henry, T. J., et al. 2010, ApJS, 190, 1

Rickman, E. L., Ségransan, D., Hagelberg, J., et al. 2020, A\&A, 635, A203

Santerne, A., Hébrard, G., Deleuil, M., et al. 2014, A\&A, 571, A37

Schneider, G., \& Silverstone, M. 2003, ASP Conf. Ser., 291, 69

Schwarz, R., Funk, B., Zechner, R., \& Bazsó, A. 2016, MNRAS, 460, 3598

Shkolnik, E. L., Allers, K. N., Kraus, A. L., Liu, M. C., \& Flagg, L. 2017, AJ, 154,69

Sirbu, D., Thomas, S., Belikov, R., \& Bendek, E. 2017, ApJ, 849, 142

Skrutskie, M. F., Cutri, R. M., Stiening, R., et al. 2006, AJ, 131, 1163

Song, I., Zuckerman, B., \& Bessell, M. S. 2012, AJ, 144, 8

Soummer, R., Pueyo, L., \& Larkin, J. 2012, ApJ, 755, L28

Sparks, W. B., \& Ford, H. C. 2002, ApJ, 578, 543

Stetson, P. B. 1987, PASP, 99, 191

Stolker, T., Bonse, M. J., Quanz, S. P., et al. 2019, A\&A, 621, A59

Tetzlaff, N., Neuhäuser, R., \& Hohle, M. M. 2011, MNRAS, 410, 190

Thalmann, C., Desidera, S., Bonavita, M., et al. 2014, A\&A, 572, A91

Tokovinin, A. A. 1997, A\&AS, 124, 75

Torres, R. M., Loinard, L., Mioduszewski, A. J., \& Rodríguez, L. F. 2009, ApJ, 698,242

Udry, S., \& Santos, N. C. 2007, ARA\&A, 45, 397

Vigan, A., Moutou, C., Langlois, M., et al. 2010, MNRAS, 407, 71

Vigan, A., Patience, J., Marois, C., et al. 2012, A\&A, 544, 9

Vigan, A., Fontanive, C., Meyer, M., et al. 2020, A\&A, in press, https: //doi . org/10.1051/0004-6361/202038107

Walt, S. v. d., Colbert, S. C., \& Varoquaux, G. 2011, Comput. Sci. Eng., 13, 22

Wang, J., Xie, J.-W., Barclay, T., \& Fischer, D. A. 2014, ApJ, 783, 4

Wang, J., Fischer, D. A., Horch, E. P., \& Xie, J.-W. 2015, ApJ, 806, 248

Wilking, B., Gagne, M., \& Allen, L. 2008, Handbook of Star Forming Regions, Volume II, ed. B. Reipurth (San Francisco: ASP), 5, 351

Winters, J. G., Henry, T. J., Jao, W.-C., et al. 2019, AJ, 157, 216

Zsom, A., Sándor, Z., \& Dullemond, C. P. 2011, A\&A, 527, A10 


\section{Appendix A: Additional material}
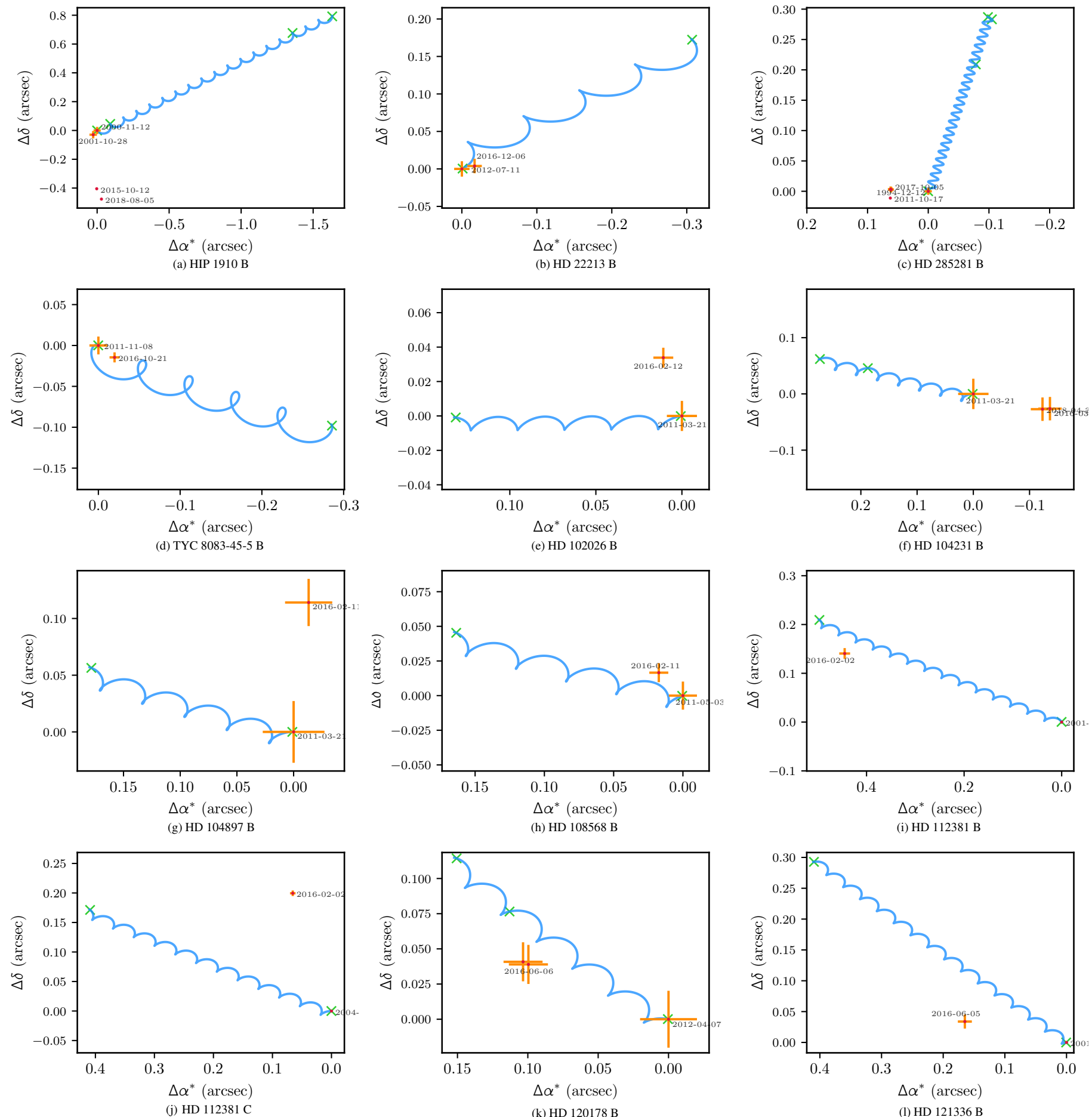

Fig. A.1. Astrometric plots of the binaries. Oranges crosses represent the relative position of the companion where the origin is defined by the first observation. The blue corkscrew line is the track a background object would follow due to the primary star proper motion with the green crosses along that track representing the positions it would have at the dates of observation. 
A\&A 643, A98 (2020)
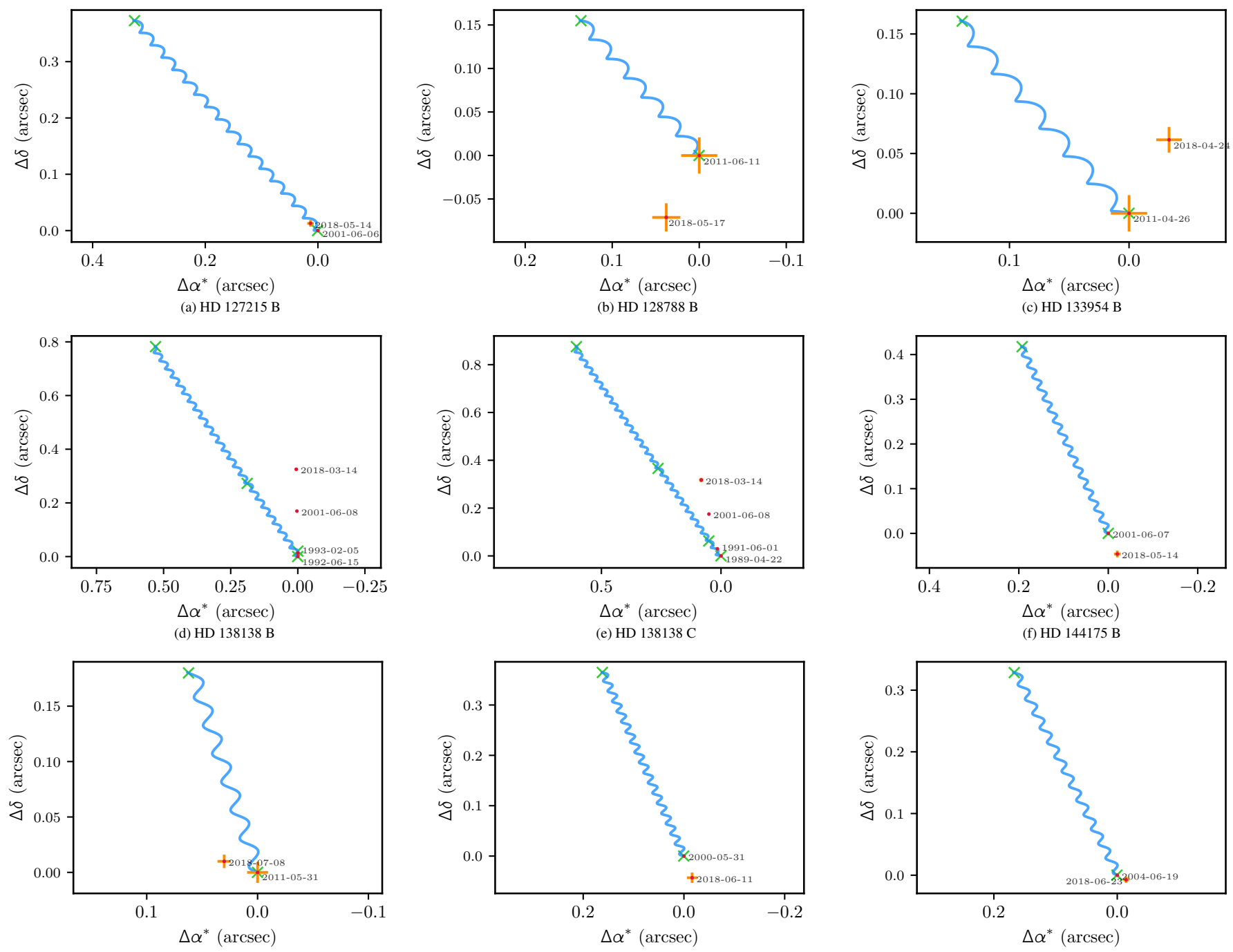

(e) HD $138138 \mathrm{C}$
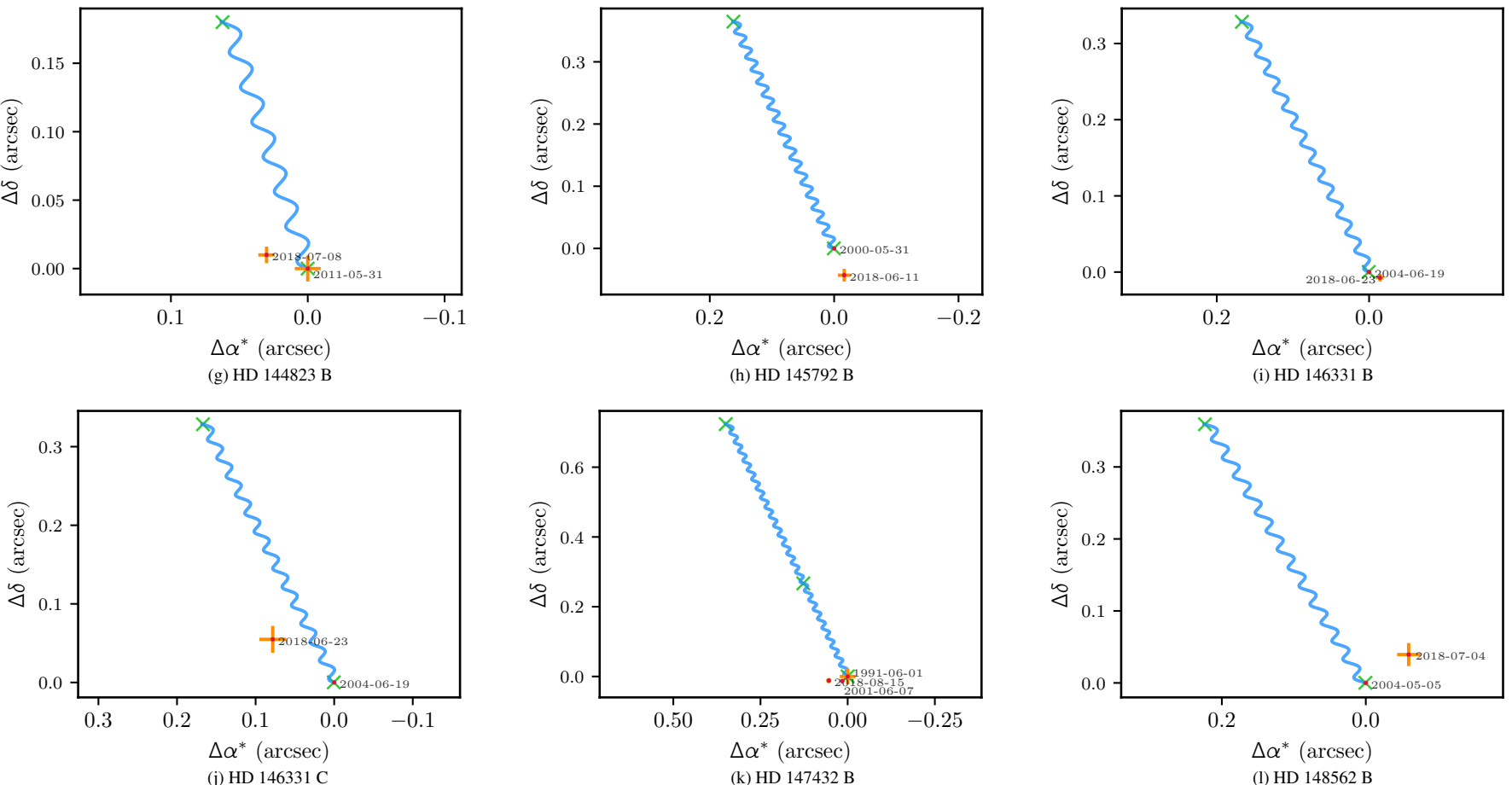

Fig. A.1. continued. 
J. Hagelberg et al.: VIBES: Visual Binary Exoplanet survey with SPHERE
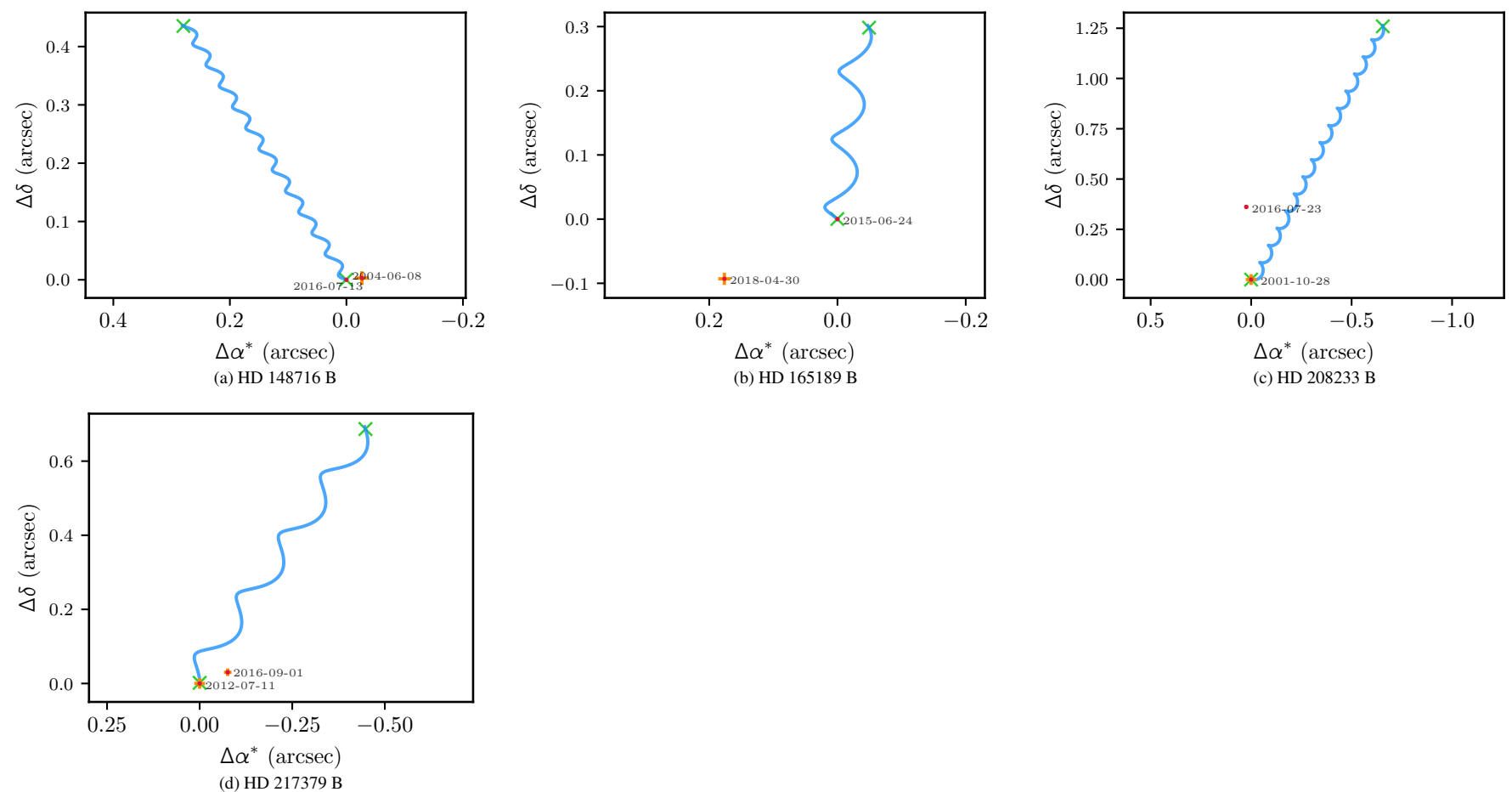

Fig. A.1. continued. 


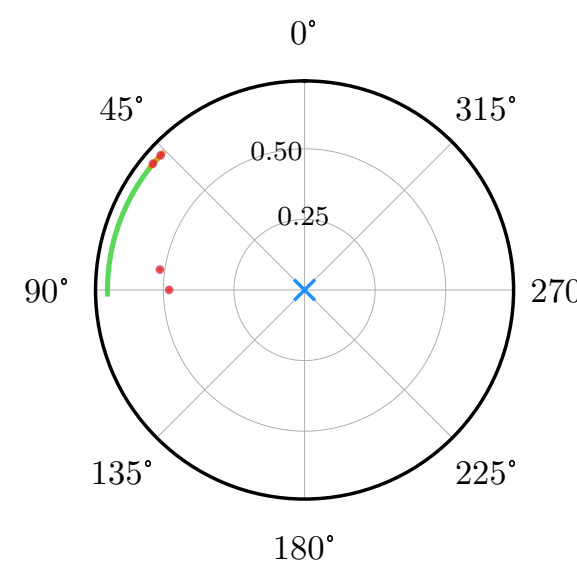

(a) HIP $1910 \mathrm{~B}$

$0^{\circ}$

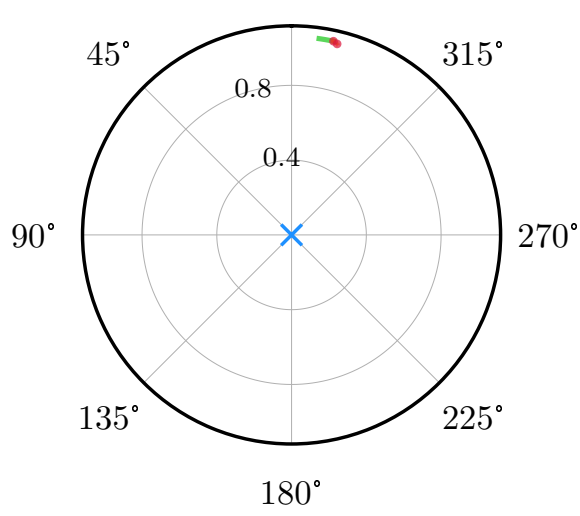

(d) TYC 8083-45-5 B

$0^{\circ}$

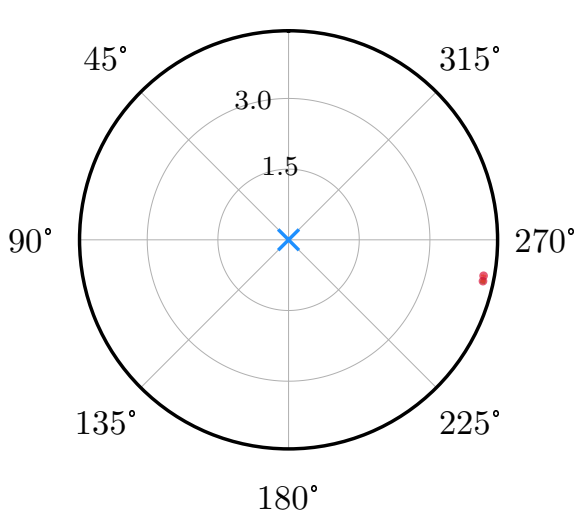

(g) HD 104897 B

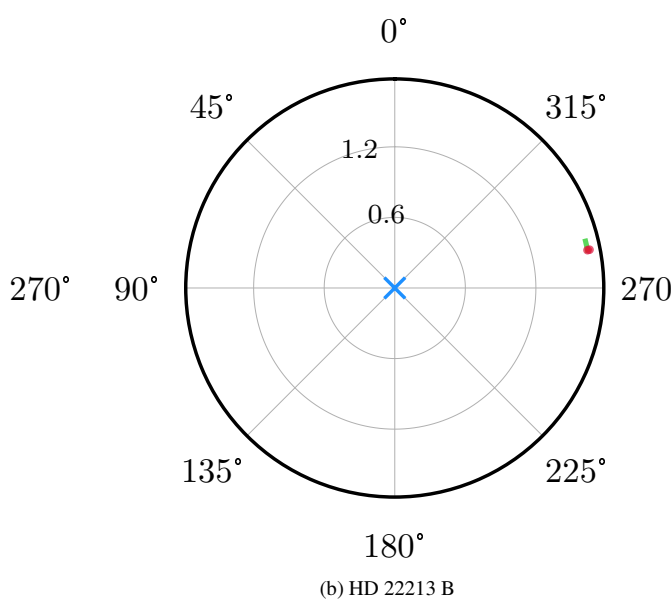

$0^{\circ}$

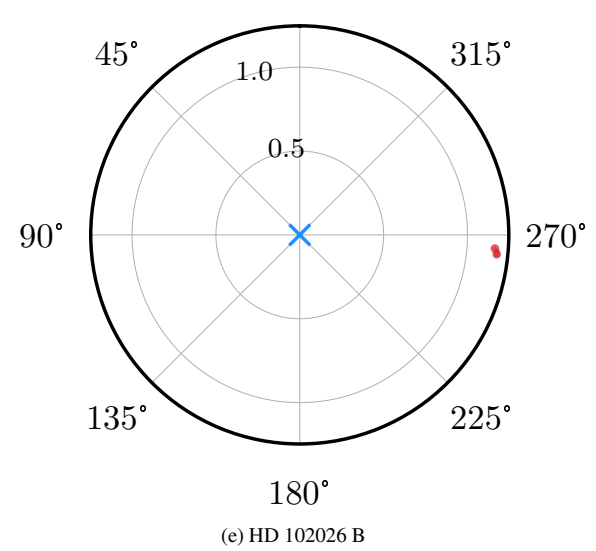

$0^{\circ}$

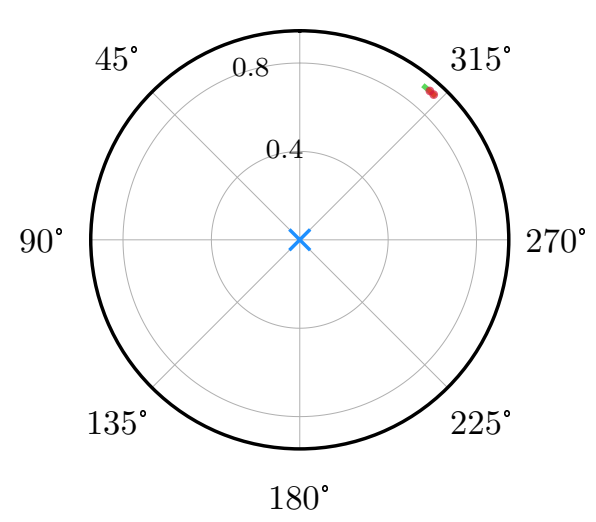

(h) HD $108568 \mathrm{~B}$

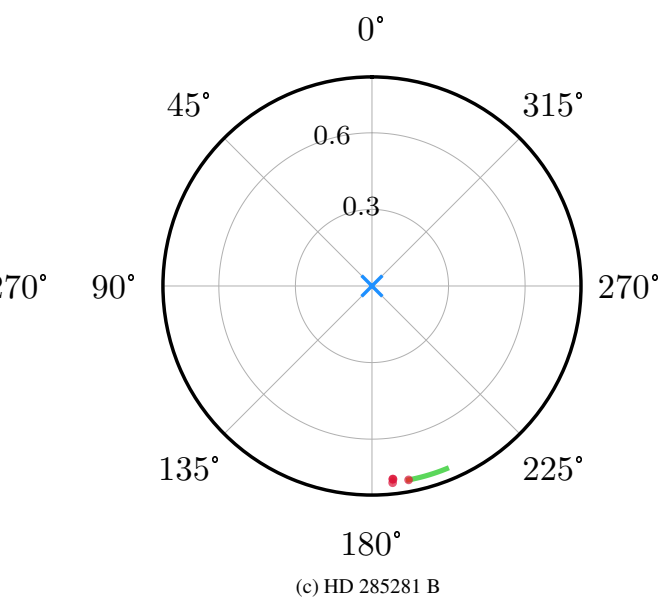

$0^{\circ}$

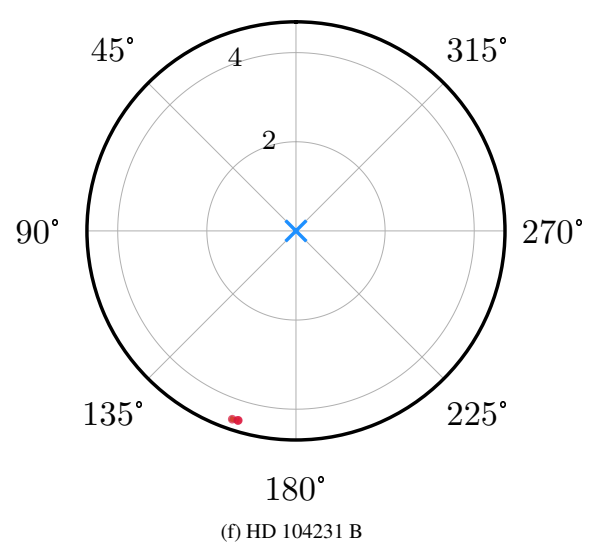

$0^{\circ}$

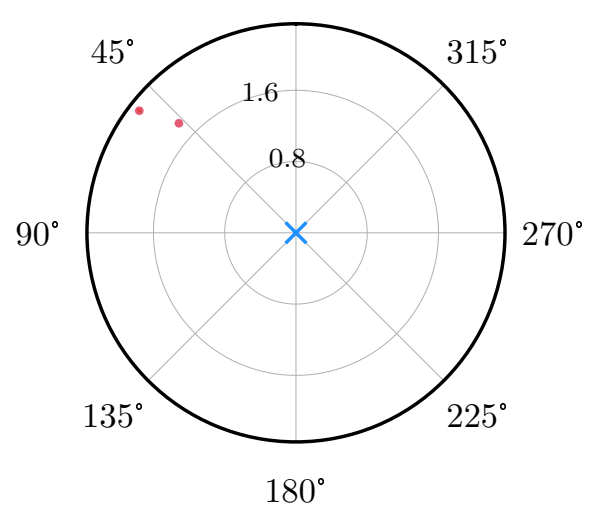

(i) HD 112381 B

Fig. A.2. Position measurements of the companions from this work and literature. The blue cross in the centre represents the primary star position while the red dots show the companion position. The green arc represents the movement a face-on circular orbit would have with the observed time baseline. Error bars are too small to be visible in the plots. 
J. Hagelberg et al.: VIBES: Visual Binary Exoplanet survey with SPHERE

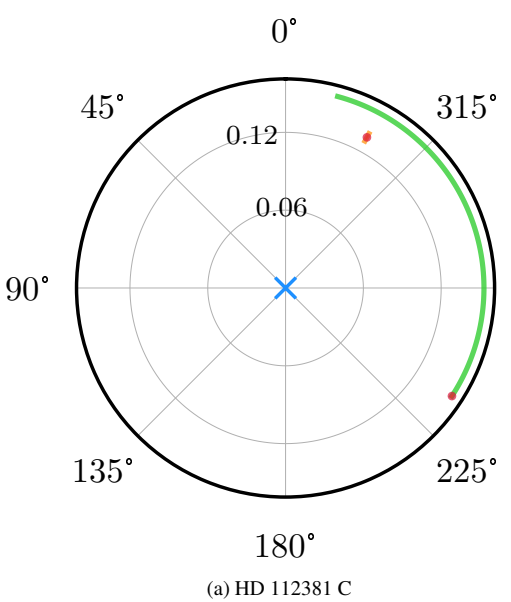

$0^{\circ}$

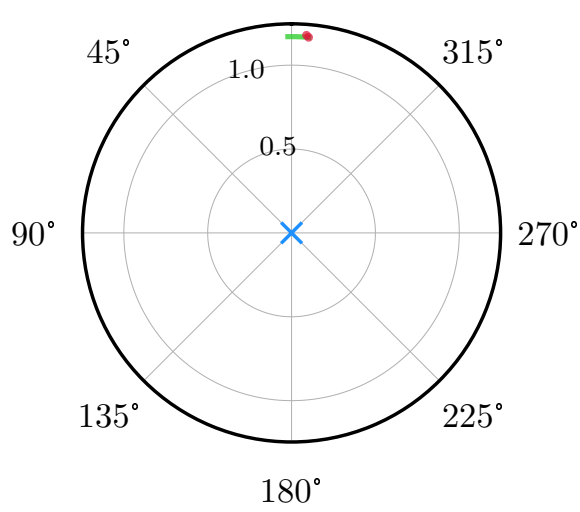

(d) HD 127215 B

$0^{\circ}$

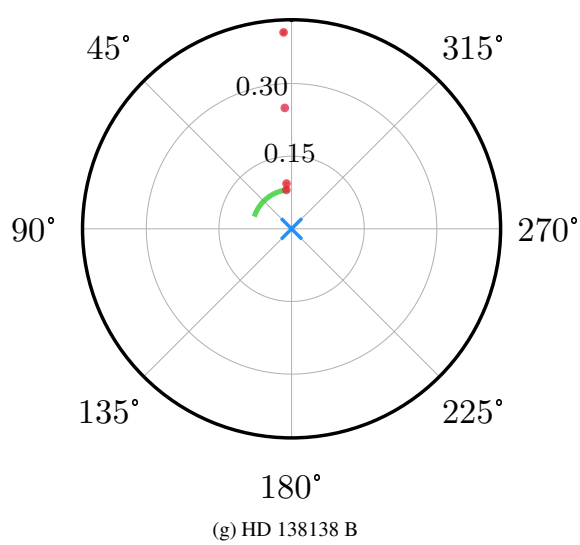

Fig. A.2. continued.

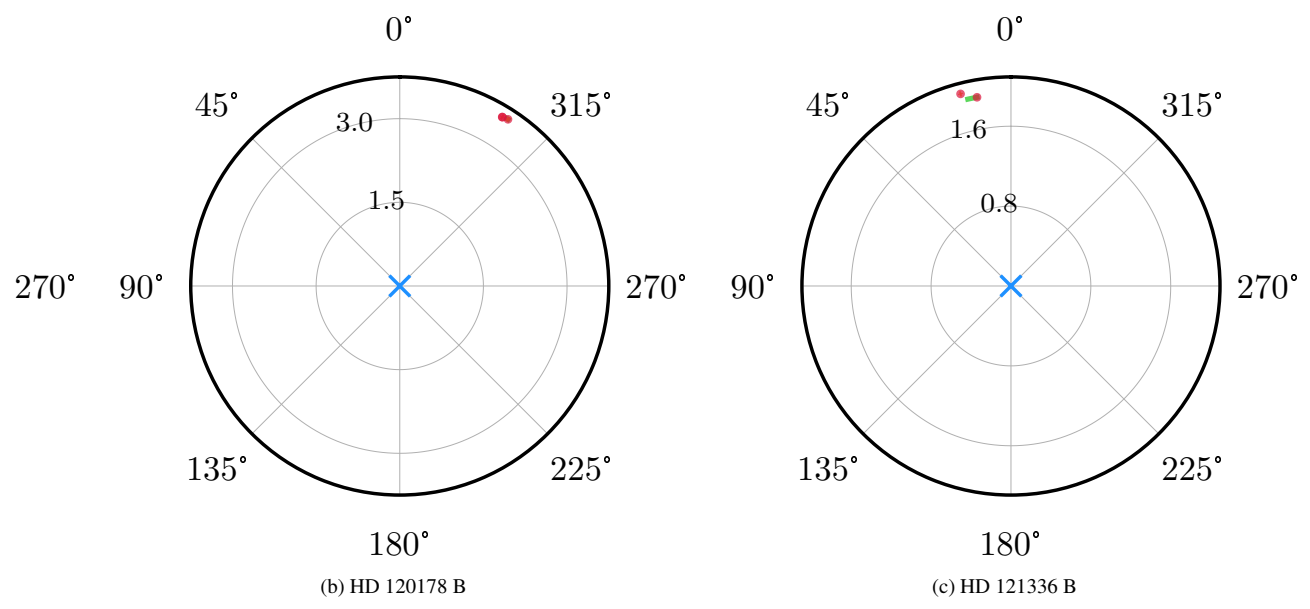

$0^{\circ}$

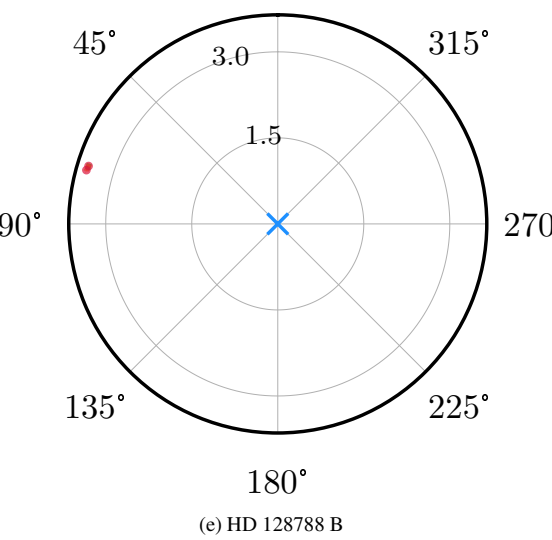

$0^{\circ}$

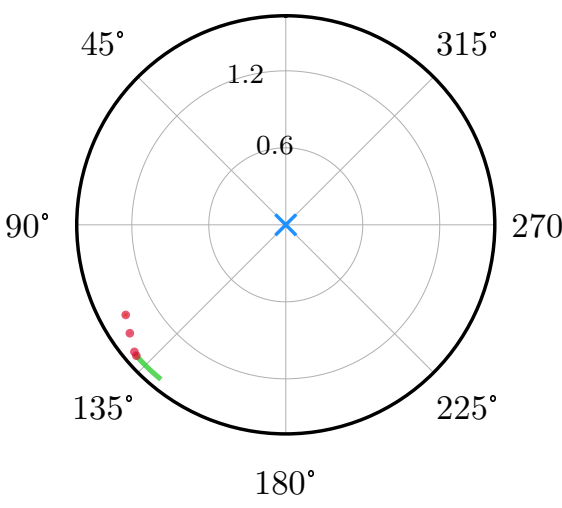

(h) HD $138138 \mathrm{C}$

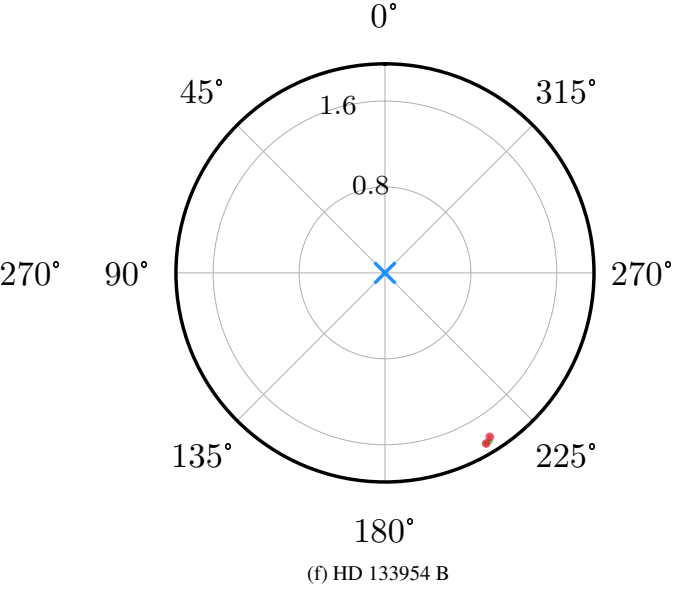

$0^{\circ}$

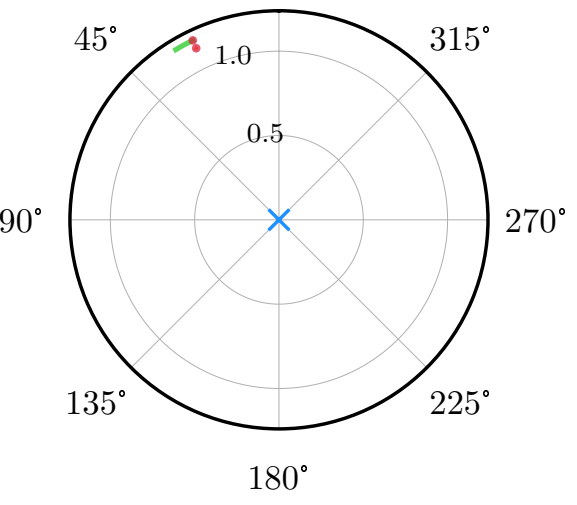

(i) HD $144175 \mathrm{~B}$ 

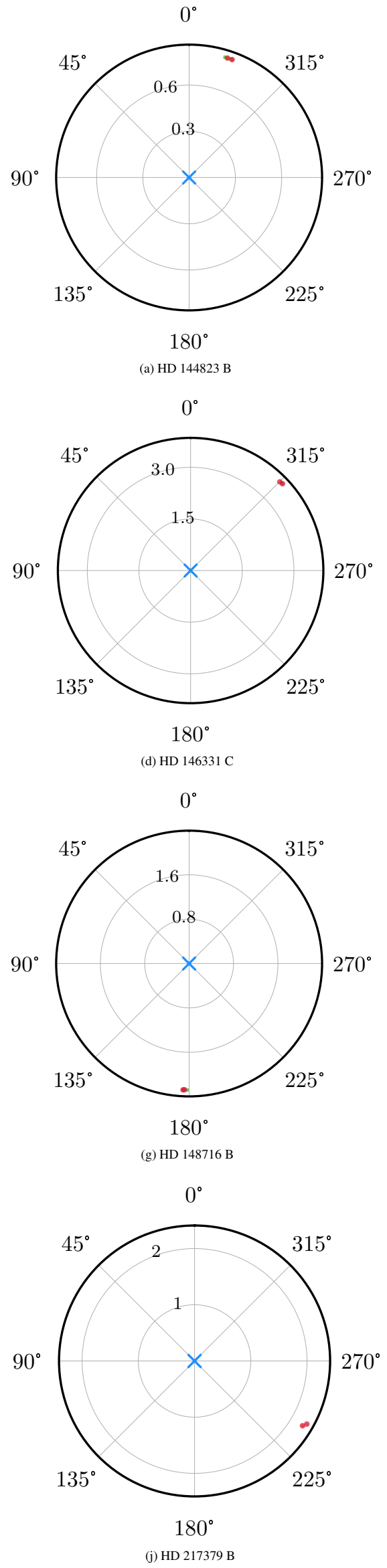

Fig. A.2. continued.

A98, page 14 of 19
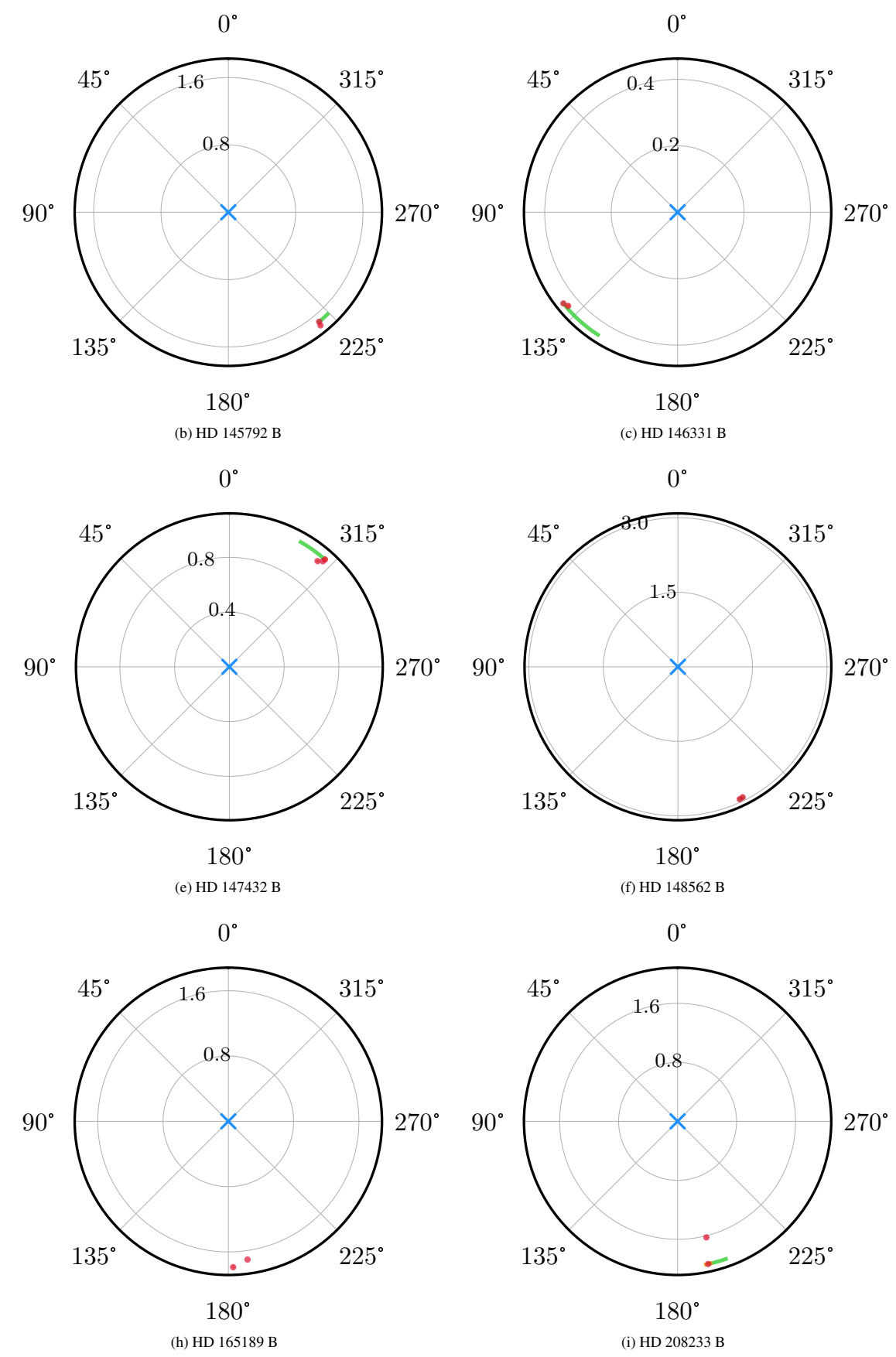

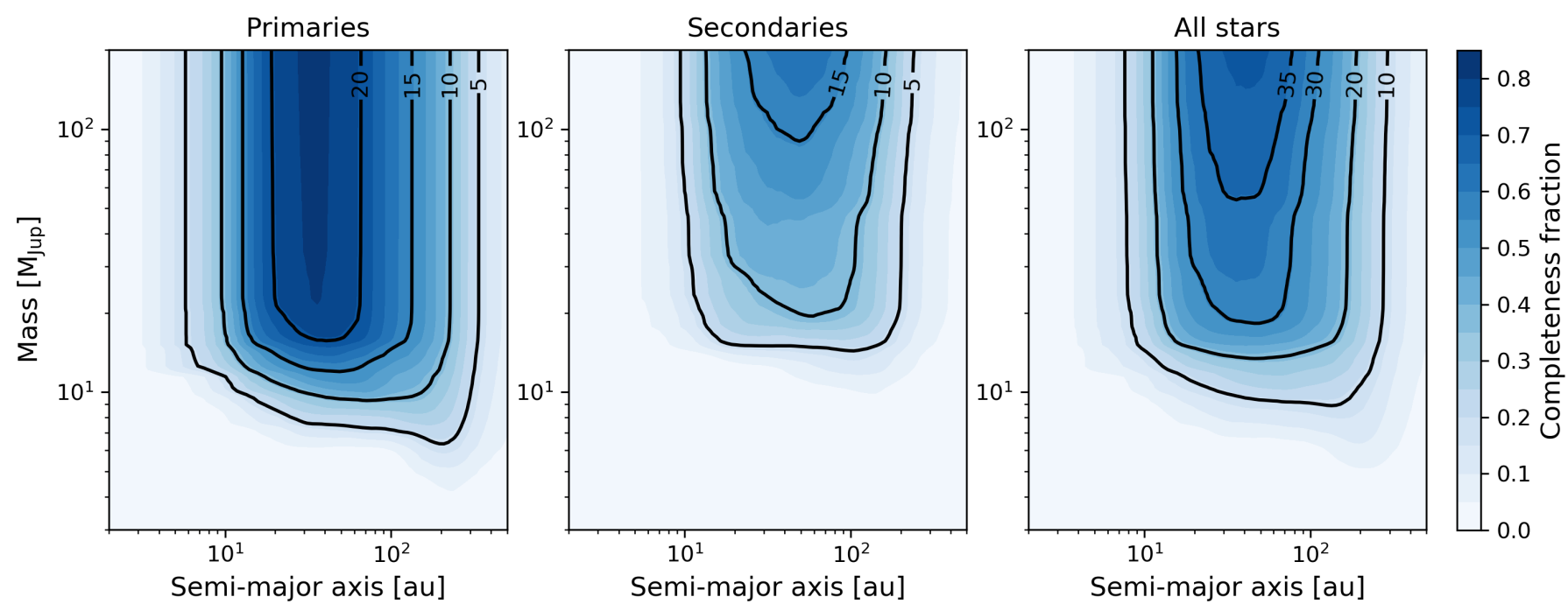

Fig. A.3. Average completeness of the survey considering the primary stars only (left panel), secondaries only (centre) and all stars together (right). The colourmap is the same in all panels and indicates the average completeness level as a function of mass and semi-major axis for each sub-sample. The black contours show the number of stars from each subset around which companions of given masses and separations are detectable, and are therefore out of 27 stars for the left and middle panels, and out of 54 targets for the right panel.
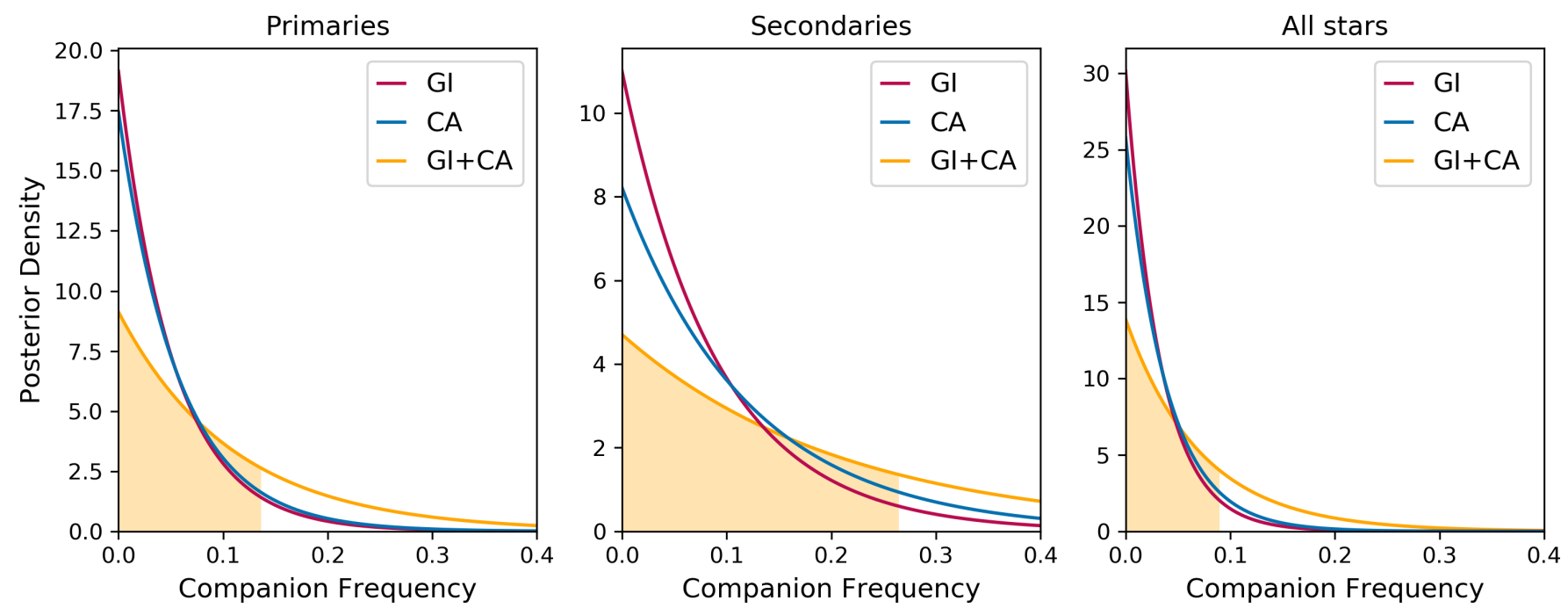

Fig. A.4. Posterior probability density functions of the frequencies of sub-stellar companions with masses in the range 10-75 $M_{\mathrm{Jup}}$ and semi-major axes between 10-200 au around the primary stars (left), secondary stars (centre) and all binary components (right). Each plot shows the posteriors obtained from the MCMC analysis for the relative frequencies of the two parts of the model population (GI in red and CA in blue), as well as for the total frequency for the full model (GI + CA in yellow). The shaded yellow areas mark the intervals of $68 \%$ confidence derived on the total frequencies of sub-stellar companions in the considered ranges. 
Table A.1. Gaia distance, coordinates, and spectral types, and probability of association or moving group (MG) for each target using the Banyan $\Sigma$ tool.

\begin{tabular}{|c|c|c|c|c|c|c|}
\hline ID & RA & Dec & SpT & $\begin{array}{l}\text { Distance } \\
\text { (pc) }\end{array}$ & $\begin{array}{c}\text { Banyan } \Sigma \\
\text { MG }\end{array}$ & $\begin{array}{c}\text { Banyan } \Sigma \text { Prob } \\
(\%)\end{array}$ \\
\hline HIP 1910 & $00: 24: 08.9$ & $-62: 11: 04.3$ & MOVe & $44.23_{-1.04}^{+1.09}$ & THA & 58.0 \\
\hline HD 22213 & $03: 34: 16.3$ & $-12: 04: 07.2$ & G8V & $51.57_{-0.16}^{+0.16}$ & THA & 98.9 \\
\hline HD 102026 & $11: 44: 09.7$ & $-53: 44: 54.3$ & A1V & $219.07_{-2.21}^{+2.26}$ & FIELD & 99.9 \\
\hline HD 104231 & 12:00:09.4 & $-57: 07: 01.9$ & F5V & $102.73_{-0.47}^{+0.47}$ & LCC & 99.9 \\
\hline HD 104897 & $12: 04: 44.4$ & $-52: 21: 15.6$ & F3V & $106.96_{-0.45}^{+0.46}$ & LCC & 97.7 \\
\hline HD 108568 & $12: 28: 40.0$ & $-55: 27: 19.3$ & G1IV & $120.44_{-0.55}^{+0.56}$ & $\mathrm{LCC}$ & 98.9 \\
\hline HD 112381 & $12: 56: 58.2$ & $-54: 35: 14.4$ & $\mathrm{ApSi}$ & $121.30_{-2.95}^{+3.10}$ & LCC & 97.6 \\
\hline HD 120178 & $13: 49: 09.2$ & $-54: 13: 42.3$ & F5V & $133.08_{-0.63}^{+0.63}$ & LCC/UCL & $42.1 / 49.5$ \\
\hline HD 121336 & $13: 56: 19.9$ & $-54: 07: 56.7$ & A1V & $139.86_{-1.74}^{+1.78}$ & UCL & 63.7 \\
\hline HD 127215 & $14: 31: 14.3$ & $-40: 39: 21.3$ & A1V & $145.99_{-1.72}^{+1.76}$ & UCL & 99.6 \\
\hline HD 128788 & $14: 40: 05.0$ & $-40: 54: 02.3$ & $\mathrm{~A} 5 \mathrm{~V}$ & $144.62_{-4.13}^{+4.32}$ & $\mathrm{UCL}$ & 99.8 \\
\hline HD 133954 & $15: 08: 42.5$ & $-44: 29: 04.3$ & A2/3III & $147.77_{-1.34}^{+1.37}$ & UCL & 99.8 \\
\hline HD 138138 & $15: 31: 17.2$ & $-33: 49: 11.4$ & $\mathrm{~A} 2 / 3 \mathrm{~V}$ & $123.87_{-1.39}^{+1.42}$ & UCL & 98.7 \\
\hline HD 144175 & $16: 05: 19.1$ & $-23: 40: 08.8$ & B9V & $143.72_{-2.00}^{+2.06}$ & USCO & 99.9 \\
\hline HD 144118 & $16: 05: 46.2$ & $-39: 50: 35.9$ & A5V & $129.03_{-0.95}^{+0.00}$ & UCL & 99.5 \\
\hline HD 144823 & $16: 08: 43.6$ & $-25: 22: 36.5$ & F3V & $160.38_{-1.16}^{+1.18}$ & USCO & 97.1 \\
\hline HD 145792 & $16: 13: 45.4$ & $-24: 25: 19.5$ & B6IV & $143.68_{-2.66}^{+2.76}$ & USCO & 99.6 \\
\hline HD 146331 & $16: 16: 50.6$ & $-25: 51: 46.7$ & B9V & $156.03_{-1.61}^{+1.65}$ & USCO & 99.2 \\
\hline HD 147432 & $16: 22: 51.7$ & $-23: 07: 07.5$ & A1III/IV & $131.41_{-2.99}^{+3.13}$ & USCO & 98.9 \\
\hline HD 148562 & $16: 29: 54.5$ & $-24: 58: 46.0$ & $\mathrm{~A} 2 \mathrm{~V}$ & $130.36_{-1.35}^{+1.37}$ & ROPH & 63.2 \\
\hline HD 148716 & $16: 31: 11.0$ & $-29: 59: 52.4$ & F3V & $118.15_{-1.07}^{+1.09}$ & UCL & 93.7 \\
\hline HD 165189 & $18: 06: 49.8$ & $-43: 25: 30.8$ & A6V & $44.56_{-0.32}^{-0.32}$ & BPMG & 94.0 \\
\hline HD 199143 & $20: 55: 47.7$ & $-17: 06: 51.0$ & F8V & $45.66_{-0.08}^{+0.08}$ & BPMG & 97.1 \\
\hline HD 208233 & $21: 57: 51.4$ & $-68: 12: 50.1$ & G9IV & $52.38_{-0.37}^{+0.08}$ & THA & 99.1 \\
\hline HD 217379 & 23:00:27.9 & $-26: 18: 42.7$ & $\mathrm{~K} 6.5 \mathrm{~V}$ & $30.54_{-0.05}^{+0.05}$ & $\mathrm{ABD}$ & 59.5 \\
\hline HD 285281 & 04:00:31.0 & $+19: 35: 20.9$ & K1 & $135.34_{-1.17}^{+1.19}$ & TAU & 99.2 \\
\hline TYC 8083-45-5 & 04:48:00.6 & $-50: 41: 25.6$ & K7Ve & $59.60_{-0.27}^{+0.27}$ & THA & 98.2 \\
\hline
\end{tabular}


Table A.2. Log of observations with the atmospheric conditions for each run.

\begin{tabular}{|c|c|c|c|c|c|c|c|}
\hline \multirow[b]{2}{*}{ HD ID } & \multirow[b]{2}{*}{ HIP ID } & \multirow[b]{2}{*}{ Date } & \multirow{2}{*}{$\begin{array}{c}\text { Field } \\
\text { rotation } \\
{\left[^{\circ}\right]}\end{array}$} & \multirow{2}{*}{$\begin{array}{c}\text { Exposure } \\
\text { time, IRDIS } \\
(1) \\
\left(\mathrm{s} \mathrm{min}^{-1}\right)\end{array}$} & \multicolumn{3}{|c|}{ Observing conditions on average } \\
\hline & & & & & Airmass & $\begin{array}{l}\text { Seeing } \\
\left({ }^{\prime \prime}\right)\end{array}$ & $\begin{array}{c}\text { Coherence time } \\
(\mathrm{ms})\end{array}$ \\
\hline- & 1910 & $2015-10-13$ & 12 & $8 \times 1 \times 124=992 / 16.5$ & $1.43-1.54$ & 1.18 & 1.4 \\
\hline- & 1910 & 2018-08-05 & 14 & $8 \times 26 \times 8=1664 / 27.7$ & $1.26-1.27$ & 0.97 & 3.6 \\
\hline 22213 & - & 2016-12-06 & 42 & $0.8 \times 16 \times 98=1312 / 21.9$ & $1.03-1.04$ & 0.65 & 6.5 \\
\hline 285281 & - & $2017-10-05$ & 20 & $0.8 \times 18 \times 72=1085 / 18.1$ & $1.40-1.42$ & 1.07 & 4.8 \\
\hline 285281 & - & 2017-12-08 & 19 & $0.8 \times 18 \times 80=1206 / 20.1$ & $1.41-1.46$ & 0.53 & 7.1 \\
\hline TYC 8083-45-5 & - & $2016-10-21$ & 21 & $2 \times 17 \times 48=1632 / 27.2$ & $1.12-1.13$ & 1.13 & 2.7 \\
\hline TYC 8083-45-5 & - & 2017-10-09 & 21 & $2 \times 17 \times 48=1632 / 27.2$ & $1.11-1.12$ & 0.61 & 5.2 \\
\hline 102026 & 57238 & 2016-02-12 & 22 & $32 \times 1 \times 80=2560 / 42.7$ & $1.15-1.16$ & 0.81 & 4.4 \\
\hline 104231 & 58528 & 2016-03-18 & 20 & $8 \times 1 \times 256=2048 / 34.1$ & $1.19-1.19$ & 1.08 & 2.6 \\
\hline 104231 & 58528 & $2018-04-30$ & 14 & $4 \times 28 \times 11=1232 / 20.5$ & $1.19-1.19$ & 1.14 & 5.6 \\
\hline 104897 & 58899 & 2016-02-11 & 23 & $8 \times 1 \times 256=2048 / 34.1$ & $1.13-1.14$ & 1.36 & 2.7 \\
\hline 108568 & 60885 & 2016-02-11 & 20 & $16 \times 1 \times 144=2304 / 38.4$ & $1.17-1.19$ & 1.07 & 3.3 \\
\hline 112381 & 63204 & 2016-02-02 & 21 & $1 \times 1 \times 831=831 / 13.9$ & $1.16-1.16$ & 1.73 & 2.0 \\
\hline 120178 & 67428 & 2016-06-06 & 20 & $8 \times 16 \times 17=2176 / 36.3$ & $1.15-1.15$ & 0.97 & 1.8 \\
\hline 120178 & 67428 & $2018-03-25$ & 15 & $32 \times 27 \times 2=1728 / 28.8$ & $1.15-1.16$ & 0.85 & 5.1 \\
\hline 121336 & 68080 & 2016-06-05 & 20 & $16 \times 8 \times 16=2048 / 34.1$ & $1.15-1.15$ & 0.76 & 2.8 \\
\hline 127215 & 70998 & 2018-05-14 & 32 & $16 \times 8 \times 16=2048 / 34.1$ & $1.04-1.05$ & 0.95 & 2.2 \\
\hline 128788 & 71708 & $2018-05-17$ & 32 & $4 \times 15 \times 32=1920 / 32.0$ & $1.04-1.05$ & 0.31 & 11.0 \\
\hline 133954 & 74104 & 2018-04-24 & 27 & $16 \times 9 \times 16=2304 / 38.4$ & $1.06-1.07$ & 0.48 & 9.7 \\
\hline 138138 & 76001 & 2018-03-14 & 59 & $8 \times 16 \times 32=4096 / 68.3$ & $1.01-1.02$ & 1.07 & 3.6 \\
\hline 144175 & 78809 & 2018-05-14 & 49 & $4 \times 30 \times 32=3840 / 64.0$ & $1.00-1.01$ & 1.00 & 1.8 \\
\hline 144118 & 78853 & $2018-05-15$ & 34 & $4 \times 30 \times 16=1920 / 32.0$ & $1.04-1.04$ & 0.67 & 4.1 \\
\hline 144118 & 78853 & $2019-05-26$ & 32 & $8 \times 15 \times 16=1920 / 32.0$ & $1.04-1.04$ & 1.09 & 2.2 \\
\hline 144823 & 79097 & 2018-07-08 & 6 & $8 \times 15 \times 16=1920 / 32.0$ & $1.02-1.03$ & 0.50 & 6.1 \\
\hline 145792 & 79530 & 2018-06-11 & 2 & $4 \times 13 \times 32=1664 / 27.7$ & $1.01-1.02$ & 1.11 & 1.8 \\
\hline 146331 & 79771 & $2018-06-23$ & 7 & $2 \times 15 \times 48=1440 / 24.0$ & $1.02-1.03$ & 0.70 & 3.2 \\
\hline 147432 & 80238 & 2018-08-15 & 4 & $1 \times 52 \times 32=1664 / 27.7$ & $1.02-1.04$ & 0.66 & 5.9 \\
\hline 148562 & 80799 & 2018-07-04 & 7 & $2 \times 15 \times 48=1440 / 24.0$ & $1.01-1.02$ & 1.55 & 1.7 \\
\hline 148716 & 80896 & 2016-07-13 & 47 & $4 \times 15 \times 32=1920 / 32.0$ & $1.01-1.02$ & 0.77 & 2.8 \\
\hline 165189 & - & 2016-04-30 & 28 & $2 \times 32 \times 16=1024 / 17.1$ & $1.06-1.06$ & 0.80 & 4.0 \\
\hline 165189 & - & $2018-04-30$ & 23 & $4 \times 26 \times 12=1248 / 20.8$ & $1.06-1.06$ & 0.53 & 6.3 \\
\hline 199143 & 103311 & 2019-08-26 & 46 & $2 \times 15 \times 48=1448 / 24.0$ & $1.01-1.02$ & 0.95 & 4.0 \\
\hline 208233 & 108422 & $2016-07-23$ & 14 & $16 \times 9 \times 16=2304 / 38.4$ & $1.38-1.38$ & 0.67 & 4.5 \\
\hline 217379 & 113597 & 2016-09-01 & 12 & $2 \times 16 \times 48=1536 / 25.6$ & $1.01-1.02$ & 0.66 & 6.3 \\
\hline
\end{tabular}

Notes. ${ }^{(1)}$ The exposure time of the IRDIS observation is given in a format "DIT $\times$ NDIT $\times$ NEXP $=$ Total exposure time in seconds/Total exposure time in minutes", where DIT is the detector integration time, NDIT is a number of integrations per exposure, NEXP is a number of exposures (files). The total exposure time of the IFS data is either the same as for the IRDIS data or slightly shorter depending on target. 
Table A.3. Target sample along with astrometric measurements from literature and from this work.

\begin{tabular}{|c|c|c|c|c|c|c|c|c|c|c|c|}
\hline HD & Pair & $\begin{array}{l}M_{I} \\
M_{\odot} \\
\end{array}$ & $\begin{array}{l}M_{I I} \\
M_{I I} \\
\end{array}$ & $\begin{array}{l}K_{I} \\
\text { (mag) }\end{array}$ & $\begin{array}{l}K_{I I} \\
\text { (mag) }\end{array}$ & $\begin{array}{l}H_{I} \\
(\mathrm{mag})\end{array}$ & $\begin{array}{l}H_{I I} \\
(\mathrm{mag})\end{array}$ & $\begin{array}{l}\text { Sep } \\
\left({ }^{\prime \prime}\right)\end{array}$ & $\begin{array}{l}\text { PA } \\
(\mathrm{deg})\end{array}$ & Date & Ref. \\
\hline \multirow[t]{2}{*}{ HD 102026} & $\mathrm{AB}$ & 1.47 & 0.14 & - & - & - & - & 1.18 & 264.40 & 2011-03-21 & 10 \\
\hline & $\mathrm{AB}$ & - & - & 8.33 & - & 8.35 & 12.62 & 1.17 & 266.00 & 2016-02-12 & 1 \\
\hline \multirow[t]{3}{*}{ HD 104231} & $\mathrm{AB}$ & 1.33 & 0.30 & - & - & - & - & 4.46 & 161.30 & $2011-03-21$ & 10 \\
\hline & $\mathrm{AB}$ & - & - & 7.42 & - & 7.48 & 9.90 & 4.44 & 163.07 & 2016-03-18 & 1 \\
\hline & $\mathrm{AB}$ & - & - & 7.42 & - & 7.48 & 10.26 & 4.45 & 162.91 & 2018-04-30 & 1 \\
\hline \multirow[t]{2}{*}{ HD 104897} & $\mathrm{AB}$ & 1.49 & 0.44 & 7.35 & - & - & - & 4.22 & 258.00 & 2011-03-21 & 10 \\
\hline & $\mathrm{AB}$ & - & - & - & - & 7.42 & 10.49 & 4.21 & 259.56 & 2016-02-11 & 1 \\
\hline \multirow[t]{2}{*}{ HD 108568} & $\mathrm{AB}$ & 2.47 & 0.24 & 7.29 & - & - & - & 0.89 & 317.30 & 2011-05-03 & 10 \\
\hline & $\mathrm{AB}$ & - & - & - & - & 7.41 & 11.11 & 0.89 & 318.84 & 2016-02-11 & 1 \\
\hline \multirow[t]{4}{*}{ HD 112381} & $\mathrm{AB}$ & - & - & 6.47 & 7.36 & - & - & 1.80 & 46.90 & $2001-12-31$ & 11 \\
\hline & $\mathrm{AB}$ & - & - & 6.09 & - & 6.22 & 7.27 & 2.23 & 52.08 & 2016-02-02 & 1 \\
\hline & $\mathrm{AC}$ & 2.05 & 1.06 & 6.78 & 8.40 & - & - & 0.15 & 237.00 & 2004-04-07 & 12 \\
\hline & $\mathrm{AC}$ & - & - & - & - & 6.22 & 7.81 & 0.13 & 331.62 & 2016-02-02 & 1 \\
\hline \multirow[t]{3}{*}{ HD 120178} & $\mathrm{AB}$ & 1.44 & 0.11 & 7.63 & - & - & - & 3.56 & 327.00 & 2012-04-07 & 10 \\
\hline & $\mathrm{AB}$ & - & - & - & - & 7.71 & 12.10 & 3.54 & 328.69 & 2016-06-06 & 1 \\
\hline & $\mathrm{AB}$ & - & - & - & - & 7.71 & 11.74 & 3.54 & 328.76 & $2018-03-25$ & 1 \\
\hline \multirow[t]{3}{*}{ HD 121336} & $\mathrm{AB}$ & 2.91 & 1.92 & 6.28 & 7.19 & - & - & 1.92 & 10.20 & 2001-06-05 & 12 \\
\hline & $\mathrm{AB}$ & - & - & - & - & 6.00 & 7.00 & 1.99 & 14.71 & $2016-06-05$ & 1 \\
\hline & $\mathrm{AC}$ & - & - & 5.94 & - & 6.00 & 9.31 & 1.65 & 12.56 & 2016-06-05 & 1 \\
\hline \multirow[t]{2}{*}{ HD 127215} & $\mathrm{AB}$ & 2.54 & 0.48 & 7.06 & 10.83 & - & - & 1.17 & 355.00 & 2001-06-06 & 12 \\
\hline & $\mathrm{AB}$ & - & - & 7.05 & - & 7.09 & 11.08 & 1.18 & 355.69 & $2018-05-14$ & 1 \\
\hline \multirow[t]{2}{*}{ HD 128788} & $\mathrm{AB}$ & 1.45 & 0.44 & 7.80 & - & - & - & 3.45 & 73.00 & 2011-06-11 & 10 \\
\hline & $\mathrm{AB}$ & - & - & - & - & - & - & 3.47 & 74.31 & $2018-05-17$ & 1 \\
\hline \multirow[t]{2}{*}{ HD 133954} & $\mathrm{AB}$ & 2.45 & 0.49 & 7.67 & - & - & - & 1.85 & 210.70 & 2011-04-26 & 10 \\
\hline & $\mathrm{AB}$ & - & - & - & - & 7.70 & 10.52 & 1.81 & 212.60 & 2018-04-24 & 1 \\
\hline \multirow[t]{8}{*}{ HD 138138} & $\mathrm{AB}$ & - & - & - & - & - & - & 0.08 & 7.60 & 1992-06-15 & 7 \\
\hline & $\mathrm{AB}$ & - & - & - & - & - & - & 0.09 & 6.40 & $1993-02-05$ & 7 \\
\hline & $\mathrm{AB}$ & 1.54 & 1.36 & 7.60 & 7.80 & - & - & 0.25 & 3.20 & $2001-06-08$ & 12 \\
\hline & $\mathrm{AB}$ & - & - & 6.43 & - & 6.49 & 6.72 & 0.41 & 2.33 & 2018-03-14 & 1 \\
\hline & $\mathrm{AC}$ & - & - & - & - & - & - & 1.55 & 131.20 & 1989-04-22 & 14 \\
\hline & $\mathrm{AC}$ & - & - & - & - & - & - & 1.54 & 130.00 & 1991 & 15 \\
\hline & $\mathrm{AC}$ & 1.54 & 1.36 & 7.60 & 8.20 & - & - & 1.48 & 124.80 & 2001-06-08 & 12 \\
\hline & $\mathrm{AC}$ & - & - & - & - & 6.49 & 7.20 & 1.43 & 119.37 & 2018-03-14 & 1 \\
\hline \multirow[t]{2}{*}{ HD 144118} & $\mathrm{AB}$ & 1.82 & 1.14 & 7.50 & 8.45 & - & - & 1.99 & 270.39 & 2001-06-08 & 12 \\
\hline & $\mathrm{AB}$ & - & - & - & - & 7.15 & 8.29 & 2.01 & 271.01 & $2018-05-15$ & 1 \\
\hline \multirow[t]{2}{*}{ HD 144175} & $\mathrm{AB}$ & 2.03 & 0.30 & 7.51 & 10.26 & - & - & 1.18 & 25.67 & 2001-06-07 & 12 \\
\hline & $\mathrm{AB}$ & - & - & 7.35 & - & 7.37 & 10.28 & 1.13 & 25.76 & 2018-05-14 & 1 \\
\hline \multirow[t]{2}{*}{ HD 144823} & $\mathrm{AB}$ & 1.99 & 0.75 & 7.25 & - & - & - & 0.81 & 340.00 & 2011-05-31 & 10 \\
\hline & $\mathrm{AB}$ & - & - & - & - & 7.33 & 10.44 & 0.81 & 342.23 & 2018-07-08 & 1 \\
\hline \multirow[t]{2}{*}{ HD 145792} & $\mathrm{AB}$ & 3.73 & 1.58 & 6.60 & 8.34 & - & - & 1.69 & 219.66 & $2000-05-31$ & 12 \\
\hline & $\mathrm{AB}$ & - & - & 7.11 & - & 6.12 & 8.16 & 1.73 & 219.16 & 2018-06-11 & 1 \\
\hline \multirow[t]{4}{*}{ HD 146331} & $\mathrm{AB}$ & 2.14 & 0.19 & 7.10 & 11.42 & - & - & 0.44 & 128.59 & 2004-06-19 & 12 \\
\hline & $\mathrm{AB}$ & - & - & 7.10 & - & 7.19 & 11.64 & 0.43 & 130.54 & $2018-06-23$ & 1 \\
\hline & $\mathrm{AC}$ & 2.14 & 0.19 & 7.10 & 10.89 & - & - & 3.67 & 313.38 & 2004-06-19 & 12 \\
\hline & $\mathrm{AC}$ & - & - & - & - & 7.19 & 11.12 & 3.65 & 314.85 & $2018-06-23$ & 1 \\
\hline \multirow[t]{3}{*}{ HD 147432} & $\mathrm{AB}$ & - & - & - & - & - & - & 1.05 & 318.30 & 1991 & 5 \\
\hline & $\mathrm{AB}$ & 1.94 & 1.67 & 7.34 & 7.49 & - & - & 1.03 & 318.46 & 2001-06-07 & 12 \\
\hline & $\mathrm{AB}$ & - & - & 6.62 & - & 6.69 & 6.91 & 1.01 & 320.15 & $2018-08-15$ & 1 \\
\hline HD 148562 & $\mathrm{AB}$ & 1.86 & 0.34 & 7.45 & 9.80 & - & - & 2.94 & 205.02 & $2004-05-05$ & 12 \\
\hline & $\mathrm{AB}$ & - & - & 7.31 & - & 7.41 & 10.92 & 2.93 & 206.40 & 2018-07-04 & 1 \\
\hline HD 148716 & $\mathrm{AB}$ & 1.81 & 0.24 & 7.44 & 10.33 & - & - & 2.28 & 177.23 & 2004-06-08 & 12 \\
\hline & $\mathrm{AB}$ & - & - & 7.45 & - & 7.55 & 11.28 & 2.28 & 177.89 & 2016-07-13 & 1 \\
\hline
\end{tabular}

Notes. The subscript A always stands for the primary star while B stands for either the secondary or tertiary component. Uncertainties reported in Table 1.

References. (1) This work; (2) Chauvin et al. (2003); (3) Daemgen et al. (2015); (4) Elliott et al. (2015); (5) Fabricius et al. (2002); (6) Gaia Collaboration (2018); (7) Hartkopf et al. (1996); (8) Herschel et al. (1874); (9) Jayawardhana \& Brandeker (2001); (10) Janson et al. (2013); (11) Kouwenhoven et al. (2005); (12) Kouwenhoven (2006); (13) Kohler \& Leinert (1998); (14) McAlister et al. (1990); (15) Tokovinin (1997). 
Table A.3. continued.

\begin{tabular}{llllllllllll}
\hline \hline HD & Pair & $\begin{array}{l}M_{I} \\
M_{\odot}\end{array}$ & $\begin{array}{l}M_{I I} \\
M_{I I}\end{array}$ & $\begin{array}{l}K_{I} \\
(\mathrm{mag})\end{array}$ & $\begin{array}{l}K_{I I} \\
(\mathrm{mag})\end{array}$ & $\begin{array}{l}H_{I} \\
(\mathrm{mag})\end{array}$ & $\begin{array}{l}H_{I I} \\
(\mathrm{mag})\end{array}$ & $\begin{array}{l}\text { Sep } \\
\left({ }^{\prime \prime}\right)\end{array}$ & $\begin{array}{l}\text { PA } \\
(\mathrm{deg})\end{array}$ & Date & Ref. \\
\hline HD 165189 & AB & - & - & 4.40 & - & - & - & 1.80 & 249.00 & 1874 & 8 \\
& AB & - & - & - & - & - & - & 1.71 & 187.91 & $2015-06-24$ & 6 \\
HD 199143 & AB & - & - & - & - & - & - & 1.79 & 181.89 & $2018-04-30$ & $\mathbf{1}$ \\
& AB & 1.19 & 0.35 & 5.9 & 8.12 & - & - & 1.08 & 325.00 & $2001-05-31$ & 9 \\
HD 208233 & AB & - & - & - & - & 5.95 & 8.19 & 0.84 & 321.71 & $2019-08-26$ & $\mathbf{1}$ \\
& AB & 1.03 & 0.28 & 6.83 & 9.78 & - & - & 1.98 & 192.10 & $2001-10-28$ & 2 \\
HD 217379 & AB & - & - & 6.83 & - & 6.86 & 9.82 & 1.62 & 193.94 & $2016-07-23$ & $\mathbf{1}$ \\
& AB & - & - & 6.75 & 7.38 & - & - & 2.24 & 239.02 & $2012-07-11$ & 4 \\
HD 22213 & AB & - & - & - & - & 6.45 & 7.31 & 2.29 & 240.64 & $2016-09-01$ & $\mathbf{1}$ \\
& AB & 0.94 & 0.54 & 6.97 & 5.10 & - & - & 1.67 & 281.10 & $2012-07-11$ & 4 \\
& AB & - & - & - & - & 6.95 & 8.84 & 1.69 & 281.12 & $2016-12-06$ & $\mathbf{1}$ \\
& AB & - & - & 7.61 & - & - & - & 0.77 & 190.70 & $1994-12-12$ & 13 \\
& AB & 1.11 & 0.71 & 7.61 & - & - & - & 0.78 & 186.00 & $2011-10-17$ & 3 \\
HIP 1910 & AB & - & - & - & - & 7.75 & 8.99 & 0.76 & 186.22 & $2017-10-05$ & $\mathbf{1}$ \\
& AB & - & - & - & - & 7.75 & 9.45 & 0.76 & 186.15 & $2017-12-08$ & $\mathbf{1}$ \\
& AB & 0.71 & 0.30 & 7.69 & 9.22 & - & - & 0.70 & 46.90 & $2000-11-12$ & 2 \\
& AB & - & - & 7.69 & - & - & - & 0.70 & 50.20 & $2001-10-28$ & 2 \\
& AB & - & - & 7.69 & - & 7.71 & 9.37 & 0.52 & 81.98 & $2015-10-12$ & $\mathbf{1}$ \\
& AB & - & - & 7.69 & - & 7.71 & 9.32 & 0.48 & 89.96 & $2018-08-05$ & $\mathbf{1}$ \\
& AB & 0.70 & 0.35 & 8.25 & 7.10 & - & - & 1.06 & 347.80 & $2011-11-08$ & 4 \\
& AB & - & - & - & - & 8.08 & 9.24 & 1.05 & 346.58 & $2016-10-21$ & $\mathbf{1}$ \\
\hline
\end{tabular}

